\title{
MACDONALD-KOORNWINDER MOMENTS AND THE TWO-SPECIES EXCLUSION PROCESS
}

\author{
SYLVIE CORTEEL AND LAUREN K. WILLIAMS
}

\begin{abstract}
Introduced in the late 1960's [MGP68, Spi70, the asymmetric exclusion process (ASEP) is an important model from statistical mechanics which describes a system of interacting particles hopping left and right on a one-dimensional lattice with open boundaries. It has been known for awhile that there is a tight connection between the partition function of the ASEP and moments of Askey-Wilson polynomials [USW04, CW11, CSSW12, a family of orthogonal polynomials which are at the top of the hierarchy of classical orthogonal polynomials in one variable. On the other hand, Askey-Wilson polynomials can be viewed as a specialization of the multivariate Macdonald-Koornwinder polynomials (also known as Koornwinder polynomials), which in turn give rise to the Macdonald polynomials associated to any classical root system via a limit or specialization vD95. In light of the fact that Koornwinder polynomials generalize the Askey-Wilson polynomials, it is natural to ask whether one can find a particle model whose partition function is related to Koornwinder polynomials. In this article we answer this question affirmatively, by showing that Koornwinder moments at $q=t$ are closely connected to the partition function for the two-species exclusion process.
\end{abstract}

\section{Contents}

1. Introduction

2. Askey-Wilson polynomials and Koornwinder polynomials at $q=t$

3. The asymmetric exclusion process and the two-species exclusion process

4. Koornwinder moments and partial Motzkin paths

5. A Jacobi-Trudi formula for general Koornwinder moments

6. From partial Motzkin paths to the two-species ASEP

7. The proof of Theorem 6.9

8. Koornwinder moments at $\xi=q=1$.

Date: February 18, 2019.

2000 Mathematics Subject Classification. Primary 05E10; Secondary 82B23, 60 C05.

Key words and phrases. Koornwinder polynomials, asymmetric exclusion process, Matrix Ansatz, staircase tableaux, Askey-Wilson polynomial.

SC was partially funded by the "Combinatoire à Paris" projet Emergences 2013-2017 and by "ALEA Sorbonne" projet IDEX USPC. LW was partially supported by the Fondation Sciences Mathématiques de Paris, the Simons foundation, a Rose-Hills Investigator award, and an NSF CAREER award. Both authors are grateful for the comments of the anonymous referees, and the support of the France-Berkeley fund, and would like to acknowledge the hospitality of LIAFA, where part of this work was carried out. 


\section{INTRODUCTION}

Introduced in the late 1960's [MGP68, Spi70], the asymmetric exclusion process (ASEP) is a model of interacting particles hopping left and right on a one-dimensional lattice of $N$ sites. In the most general form of the ASEP with open boundaries, particles may enter and exit at the left with probabilities $\alpha$ and $\gamma$, and they may exit and enter at the right with probabilities $\beta$ and $\delta$. In the bulk, the probability of hopping left is $q$ times the probability of hopping right. The ASEP is important in statistical mechanics because it is one of the simplest models which exhibits boundary-induced phase transitions. Moreover, the ASEP has been cited as a model for traffic flow and protein synthesis.

It has been known since work of Uchiyama-Sasamoto-Wadati [USW04] that there is a close connection between the partition function $Z_{N}$ of the ASEP, and AskeyWilson polynomials, a family of orthogonal polynomials which are at the top of the hierarchy of classical orthogonal polynomials in one variable. Using their work, we showed in CSSW12] that each Askey-Wilson moment equals a specialization of the fugacity partition function $Z_{N}(\xi)$ of the ASEP, see Theorem 4.1. In [CW11, CW13, we introduced some new combinatorial objects called staircase tableaux, and used them to completely describe the stationary distribution of the ASEP; in particular, the partition function $Z_{N}(\xi)$ can be written as a sum over staircase tableaux of size $N$. It follows that (up to a scalar factor), $Z_{N}(\xi)$ is a polynomial with positive coefficients, and that Askey-Wilson moments can be expressed in terms of staircase tableaux.

Askey-Wilson polynomials can be viewed as a specialization of the multivariate Macdonald-Koornwinder polynomials, which are also known as Koornwinder polynomials [Koo92], or Macdonald polynomials for the type BC root system [Mac95]. (For brevity, we will henceforth call them Koornwinder polynomials.) These polynomials are particularly important because the Macdonald polynomials associated to any classical root system can be expressed as limits or special cases of Koornwinder polynomials vD95]. Since Koornwinder polynomials generalize Askey-Wilson polynomials, and Askey-Wilson moments are closely connected to the ASEP, it is natural to ask if there is some particle model generalizing the ASEP whose partition function is related to Koornwinder polynomials. This question was posed to us by Mark Haiman in 2007 [Hai].

When $q=t$, Koornwinder polynomials can be expressed in terms of Askey-Wilson

polynomials by means of a Schur-like determinantal formula. This fact, together with the connection between Askey-Wilson moments and the partition function $Z_{N}(\xi)$ of the ASEP, led Eric Rains [Rai] to suggest that we consider, for any partition 
$\lambda=\left(\lambda_{1}, \lambda_{2}, \ldots, \lambda_{m}\right)$, the quantity

$$
K_{\lambda}(\xi)=\frac{\operatorname{det}\left(Z_{\lambda_{i}+m-i+m-j}(\xi)\right)_{i, j=1}^{m}}{\operatorname{det}\left(Z_{2 m-i-j}(\xi)\right)_{i, j=1}^{m}} .
$$

The $K_{\lambda}(\xi)$ 's can be considered to be Koornwinder moments, see Sections 2 and 4.1 .

In this paper we will answer Haiman's question affirmatively by demonstrating that there is a close connection between Koornwinder moments and the partition function of the two-species ASEP. The two-species ASEP is a generalization of the ASEP which involves two different kinds of particles, "heavy" and "light". Both types of particles can hop left and right in the lattice (heavy and light particles interact exactly as do particles and holes in the usual ASEP), but only the heavy particles can enter and exit the lattice at the left and right boundary. So in particular, the number of light particles is conserved. When there are no light particles, the two-species ASEP reduces to the usual ASEP. The main result of this paper is an interpretation of the "complete homogeneous" Koornwinder moments $K_{(m, 0,0, \ldots, 0)}(\xi)$ in terms of the partition function of the two-species ASEP. That is, we prove the following.

Theorem 1.1. The Koornwinder moment $K_{(N-r, 0,0, \ldots, 0)}(\xi)$ (where there are precisely $r 0$ 's in the partition) is proportional to the fugacity partition function $Z_{N, r}(\xi)$ for the two-species ASEP on a lattice of $N$ sites with $r$ "light" particles. More specifically,

$$
K_{(N-r, 0,0, \ldots, 0)}(\xi)=Z_{N, r}(\xi) .
$$

As an intermediate step towards proving Theorem 1.1, we give a combinatorial interpretation of $Z_{N, r}(\xi)$ in terms of partial Motzkin paths, see Theorem 6.1. This leads to an integral representation for $Z_{N, r}(\xi)$, see Corollary 6.2, which in turn can be used to compute asymptotics.

We also prove a Jacobi-Trudi-type formula which expresses any arbitrary Koornwinder moment $K_{\lambda}$ as a determinant in the complete homogeneous Koornwinder moments, see Corollary 5.2 .

Since the partition function $Z_{N}$ is a polynomial with positive coefficients [CW11, CW13, Rains conjectured that the Koornwinder moments $K_{\lambda}$ are also polynomials with positive coefficients (up to a simple scalar factor) Rai]. Note that by the probabilistic interpretation of Theorem 1.1, it follows that $K_{(N-r, 0,0, \ldots, 0)}$ is positive whenever we specialize the parameters $\alpha, \beta, \gamma, \delta$, and $q$ to be positive numbers between 0 and 1. Moreover, in a sequel to this paper [CMW17], we will give a tableaux formula for $Z_{N, r}$, which implies that both $Z_{N, r}$ and $K_{(N-r, 0,0, \ldots, 0)}$ are (up to a scalar factor) polynomials in the parameters with positive coefficients.

It is worth noting that there has been some recent work which may be related to ours. In [BC14], Borodin and Corwin introduced what they call Macdonald processes, which are probability measures on sequences of partitions defined in terms of nonnegative specializations of the (type A) Macdonald symmetric functions, and 
showed that they are related to various interacting particle systems. None of the particle systems they discuss is the ASEP (or two-species ASEP) with open boundaries, but perhaps there exists some more general Koornwinder processes which would be connected to the ASEP.

Additionally, while we were writing up this paper, we learned about some work of Cantini [Can17], which contains a result similar to our Theorem 1.1. However, Cantini uses the partition $\left(1^{N-r}, 0^{r}\right)$, and his techniques are completely different from ours; e.g. he uses the affine Hecke algebra of type $\hat{C}_{N}$ as opposed to the Matrix Ansatz and the combinatorics of Motzkin paths.

The structure of this paper is as follows. In Section 2, we give an introduction to Askey-Wilson polynomials, and Koornwinder polynomials, and Koornwinder moments at $q=t$. In Section 3 we define the asymmetric exclusion process and the two-species exclusion process. We also explain the Matrix Ansatz, which is a powerful tool for analyzing the stationary distribution of these models. In Section 4, we give an interpretation of Koornwinder moments in terms of weighted Motzkin paths. In Section 5 we state and prove a Jacobi-Trudi type formula for Koornwinder moments. In Section 6 we prove a connection between partial Motzkin paths and the two-species exclusion process. In Section 7, we complete the proof of our main result. Finally in Section 8 we show that when we specialize $\xi=q=1$, the Koornwinder moments $K_{\lambda}$ have a beautiful multiplicative formula in terms of the hook lengths of the corresponding partition. This formula provides evidence for the positivity conjecture regarding Koornwinder moments.

ACKnowledgments: We would like to thank Mark Haiman, who pointed out to us in 2007 that Koornwinder polynomials generalize the Askey-Wilson polynomials, and asked us if we could make a connection between Koornwinder polynomials and some generalization of the ASEP. We would also like to thank Eric Rains, who suggested that we look at Koornwinder polynomials for $q=t$, and in particular at the quantities defined by (11). Finally we would like to thank Jennifer Morse and Dennis Stanton for useful comments, and Donghyun Kim for noticing a typo in Theorem 1.1 in the published version of the paper (which is corrected in this version).

\section{Askey-Wilson polynomials and Koornwinder polynomials at $q=t$}

The Askey-Wilson polynomials are orthogonal polynomials with five free parameters $(a, b, c, d, q)$. They reside at the top of the hierarchy of the one-variable orthogonal polynomial family in the Askey scheme [AW85, GR04, KLS10a. In this section we define Askey-Wilson polynomials, following the exposition of [AW85] and [USW04, as well as Askey-Wilson moments. We will then define Koornwinder polynomials and their moments for $q=t$.

The $q$-shifted factorial is defined by

$$
\left(a_{1}, a_{2}, \cdots, a_{s} ; q\right)_{n}=\prod_{r=1}^{s} \prod_{k=0}^{n-1}\left(1-a_{r} q^{k}\right)
$$


and the basic hypergeometric function is given by

$$
{ }_{r} \phi_{s}\left[\begin{array}{l}
a_{1}, \cdots, a_{r} \\
b_{1}, \cdots, b_{s}
\end{array} ; q, z\right]=\sum_{k=0}^{\infty} \frac{\left(a_{1}, \cdots, a_{r} ; q\right)_{k}}{\left(b_{1}, \cdots, b_{s}, q ; q\right)_{k}}\left((-1)^{k} q^{k(k-1) / 2}\right)^{1+s-r} z^{k} .
$$

The Askey-Wilson polynomial $P_{n}(x)=P_{n}(x ; a, b, c, d \mid q)$ is explicitly defined by

$$
P_{n}(x)=a^{-n}(a b, a c, a d ; q)_{n}{ }_{4} \phi_{3}\left[\begin{array}{c}
q^{-n}, q^{n-1} a b c d, a e^{i \theta}, a e^{-i \theta} \\
a b, a c, a d
\end{array} ; q, q\right],
$$

with $x=\cos \theta$ for $n \in \mathcal{Z}_{+}:=\{0,1,2, \cdots\}$. It satisfies the three-term recurrence

$$
A_{n} P_{n+1}(x)+B_{n} P_{n}(x)+C_{n} P_{n-1}(x)=2 x P_{n}(x),
$$

with $P_{0}(x)=1$ and $P_{-1}(x)=0$, where

$$
\begin{aligned}
& A_{n}=\frac{1-q^{n-1} a b c d}{\left(1-q^{2 n-1} a b c d\right)\left(1-q^{2 n} a b c d\right)}, \\
& B_{n}=\frac{q^{n-1}}{\left(1-q^{2 n-2} a b c d\right)\left(1-q^{2 n} a b c d\right)}\left[\left(1+q^{2 n-1} a b c d\right)\left(q s+a b c d s^{\prime}\right)-q^{n-1}(1+q) a b c d\left(s+q s^{\prime}\right)\right], \\
& C_{n}=\frac{\left(1-q^{n}\right)\left(1-q^{n-1} a b\right)\left(1-q^{n-1} a c\right)\left(1-q^{n-1} a d\right)\left(1-q^{n-1} b c\right)\left(1-q^{n-1} b d\right)\left(1-q^{n-1} c d\right)}{\left(1-q^{2 n-2} a b c d\right)\left(1-q^{2 n-1} a b c d\right)},
\end{aligned}
$$

$$
\text { and } s=a+b+c+d, \quad s^{\prime}=a^{-1}+b^{-1}+c^{-1}+d^{-1} .
$$

Remark 2.1. It is obvious from the three-term recurrence that the polynomials $P_{n}(x)$ are symmetric in $a, b, c$ and $d$.

For $|a|,|b|,|c|,|d|<1$, using $z=e^{i \theta}$, the orthogonality is expressed by

$$
\oint_{C} \frac{d z}{4 \pi i z} w\left(\frac{z+z^{-1}}{2}\right) P_{m}\left(\frac{z+z^{-1}}{2}\right) P_{n}\left(\frac{z+z^{-1}}{2}\right)=\frac{h_{n}}{h_{0}} \delta_{m n},
$$

where the integral contour $C$ is a closed path which encloses the poles at $z=a q^{k}, b q^{k}$, $c q^{k}, d q^{k}\left(k \in \mathcal{Z}_{+}\right)$and excludes the poles at $z=\left(a q^{k}\right)^{-1},\left(b q^{k}\right)^{-1},\left(c q^{k}\right)^{-1},\left(d q^{k}\right)^{-1}$ $\left(k \in \mathcal{Z}_{+}\right)$, and where

$$
\begin{aligned}
& w(\cos \theta)=\frac{\left(e^{2 i \theta}, e^{-2 i \theta} ; q\right)_{\infty}}{\left(a e^{i \theta}, a e^{-i \theta}, b e^{i \theta}, b e^{-i \theta}, c e^{i \theta}, c e^{-i \theta}, d e^{i \theta}, d e^{-i \theta} ; q\right)_{\infty}}, \\
& \frac{h_{n}}{h_{0}}=\frac{\left(1-q^{n-1} a b c d\right)(q, a b, a c, a d, b c, b d, c d ; q)_{n}}{\left(1-q^{2 n-1} a b c d\right)(a b c d ; q)_{n}}, \\
& h_{0}=\frac{(a b c d ; q)_{\infty}}{(q, a b, a c, a d, b c, b d, c d ; q)_{\infty}} .
\end{aligned}
$$

(In the other parameter region, the orthogonality is continued analytically.)

Remark 2.2. Note that our definition of the weight function above differs slightly from the definition given in [AW85]; the weight function in [AW85] did not have the $h_{0}$ in the denominator. Our convention simplifies some of the formulas to come. 
Definition 2.3. The moments of the (weight function of the) Askey-Wilson polynomials - which we sometimes refer to as simply the Askey-Wilson moments - are defined by

$$
\mu_{k}=\mu_{k}(a, b, c, d \mid q)=\oint_{C} \frac{d z}{4 \pi i z} w\left(\frac{z+z^{-1}}{2}\right)\left(\frac{z+z^{-1}}{2}\right)^{k} .
$$

Definition 2.4. Let $\mathbf{z}=\left(z_{1}, \ldots, z_{m}\right), \lambda=\left(\lambda_{1}, \ldots, \lambda_{m}\right)$ be a partition, and $a, b, c, d, q, t$ be generic complex parameters. The Koornwinder polynomials $P_{\lambda}(\mathbf{z} ; a, b, c, d \mid q, t)$ are multivariate orthogonal polynomials which are the type BC-case of Macdonald polynomials. More specifically, $P_{\lambda}(\mathbf{z} ; a, b, c, d \mid q, t)$ is the unique Laurent polynomial which is invariant under permutation and inversion of variables, with leading monomial $\mathbf{z}^{\lambda}$, and orthogonal with respect to the Koornwinder density

$\prod_{1 \leq i<j \leq m} \frac{\left(z_{i} z_{j}, z_{i} / z_{j}, z_{j} / z_{i}, 1 / z_{i} z_{j} ; q\right)_{\infty}}{\left(t z_{i} z_{j}, t z_{i} / z_{j}, t z_{j} / z_{i}, t / z_{i} z_{j} ; q\right)_{\infty}} \prod_{1 \leq i \leq m} \frac{\left(z_{i}^{2}, 1 / z_{i}^{2} ; q\right)_{\infty}}{\left(a z_{i}, a / z_{i}, b z_{i}, b / z_{i}, c z_{i}, c / z_{i}, d z_{i}, d / z_{i} ; q\right)_{\infty}}$ on the unit torus $\left|z_{1}\right|=\left|z_{2}\right|=\cdots=\left|z_{m}\right|=1$, where the parameters satisfy $|a|,|b|,|c|,|d|,|q|,|t|<1$.

At $q=t$, we have

$$
P_{\lambda}(\mathbf{z} ; a, b, c, d \mid q, q)=\mathrm{const} \cdot \frac{\operatorname{det}\left(p_{m-j+\lambda_{j}}\left(z_{i} ; a, b, c, d \mid q\right)\right)_{i, j=1}^{m}}{\operatorname{det}\left(p_{m-j}\left(z_{i} ; a, b, c, d \mid q\right)\right)_{i, j=1}^{m}}
$$

where the $p_{i}$ 's are the Askey-Wilson polynomials.

Note that when $q=t$, the Koornwinder density becomes

$$
\prod_{1 \leq i<j \leq m}\left(1-z_{i} z_{j}\right)\left(1-z_{i} / z_{j}\right)\left(1-z_{j} / z_{i}\right)\left(1-1 / z_{i} z_{j}\right) \prod_{1 \leq i \leq m} w\left(\frac{z_{i}+z_{i}^{-1}}{2}\right),
$$

where $w$ denotes the Askey-Wilson density.

Remark 2.5. When $q=t$, Koornwinder polynomials are sometimes called Macdonald's 9th variation of Schur functions associated with Askey-Wilson polynomials [NNSY01.

For Askey-Wilson polynomials, the $k$ th moment $\mu_{k}$ is defined to be the integral of $x^{k}$ (here $x=\frac{z+z^{-1}}{2}$ ) with respect to the Askey-Wilson density. For the multivariate Koornwinder polynomials, there are several ways that we could define moments. One way would be to integrate a monomial in $x_{1}, \ldots, x_{m}$ (here we set $x_{i}=\frac{z_{i}+z_{i}^{-1}}{2}$ ) with respect to the Koornwinder density (2). Following a suggestion of Eric Rains [Rai], we will instead define our Koornwinder moments by integrating Schur polynomials $s_{\lambda}\left(x_{1}, \ldots, x_{m}\right)$ with respect to (2).

Definition 2.6. Let $I_{k}\left(f\left(x_{1}, \ldots, x_{m}\right) ; a, b, c, d ; q, q\right)$ denote the result of integrating the function $f\left(x_{1}, \ldots, x_{m}\right)$ with respect to the Koornwinder density (2). We define the Koornwinder moment

$$
M_{\lambda}=M_{\lambda}(a, b, c, d \mid q)=I_{k}\left(s_{\lambda}\left(x_{1}, \ldots, x_{m}\right) ; a, b, c, d ; q, q\right) .
$$


As Rains pointed out to us, these Koornwinder moments have a determinantal formula Rai].

Lemma 2.7. We have that

$$
M_{\lambda}=\frac{\operatorname{det}\left(\mu_{\lambda_{i}+m-i+m-j}\right)_{i, j=1}^{m}}{\operatorname{det}\left(\mu_{2 m-i-j}\right)_{i, j=1}^{m}}
$$

where $\mu_{k}$ is an Askey-Wilson moment.

Proof. Note that when $q=t$, the Koornwinder density can be written (up to a scalar factor of $\left.2^{m(m-1)}\right)$ as

$$
\prod_{1 \leq i<j \leq m}\left(x_{i}-x_{j}\right)^{2} \prod_{1 \leq i \leq m} w\left(x_{i}\right)
$$

where $x_{i}=\frac{z_{i}+z_{i}^{-1}}{2}$.

Recall that the classical definition of the Schur polynomials says that

$$
s_{\lambda}\left(x_{1}, \ldots, x_{m}\right)=\frac{\operatorname{det}\left(x_{j}^{\lambda_{i}+m-i}\right)_{i, j=1}^{m}}{\operatorname{det}\left(x_{j}^{m-i}\right)_{i, j=1}^{m}}
$$

so that

$$
s_{\lambda}\left(x_{1}, \ldots, x_{m}\right) \prod_{1 \leq i<j \leq m}\left(x_{i}-x_{j}\right)^{2}=\operatorname{det}_{1 \leq i, j \leq m}\left(x_{j}^{\lambda_{i}+m-i}\right) \underset{1 \leq i, j \leq m}{\operatorname{det}}\left(x_{j}^{m-i}\right) .
$$

Therefore we have that

$$
\begin{aligned}
M_{\lambda} & \propto \int \operatorname{det}_{1 \leq i, j \leq m}\left(x_{j}^{\lambda_{i}+m-i}\right) \operatorname{det}_{1 \leq i, j \leq m}\left(x_{j}^{m-i}\right) \prod_{1 \leq i \leq m} w\left(x_{i}\right) \\
& \propto \operatorname{det}_{1 \leq i, j \leq m} \int x^{\lambda_{i}+m-i} x^{m-j} w(x) \\
& =\operatorname{det}_{1 \leq i, j \leq m} \mu_{\lambda_{i}+m-i+m-j} .
\end{aligned}
$$

Here we obtained the second line by applying the integral version of the CauchyBinet formula dB55, And83, and we obtained the third line by using the definition of Askey-Wilson moments.

Since the constant of proportionality is independent of $\lambda$, we can recover it by setting $\lambda=0$, and using $I_{k}(1)=1$. This gives us

$$
I_{K}\left(s_{\lambda}\left(x_{1}, \ldots, x_{m}\right) ; a, b, c, d ; q, q\right)=\frac{\operatorname{det}\left(\mu_{\lambda_{i}+m-i+m-j}\right)_{i, j=1}^{m}}{\operatorname{det}\left(\mu_{2 m-i-j}\right)_{i, j=1}^{m}} .
$$




\section{The ASYMmetriC EXCLUSION PROCESS AND THE TWO-SPECIES EXCLUSION} PROCESS

We start by defining the asymmetric exclusion process (ASEP) with open boundaries. We will then define the two-species exclusion process, which generalizes the usual ASEP. Finally we will explain the Matrix Ansatz, which has been an important tool for analyzing the stationary distribution of the ASEP.

\subsection{The asymmetric exclusion process (ASEP).}

Definition 3.1. Let $\alpha, \beta, \gamma, \delta, q$, and $u$ be constants such that $0 \leq \alpha \leq 1,0 \leq \beta \leq 1$, $0 \leq \gamma \leq 1,0 \leq \delta \leq 1,0 \leq q \leq 1$, and $0 \leq u \leq 1$. Let $B_{N}$ be the set of all $2^{N}$ words in the language $\{\mathrm{o}, \bullet\}^{*}$. The ASEP is the Markov chain on $B_{N}$ with transition probabilities:

- If $X=A \bullet \circ B$ and $Y=A \circ \bullet B$ then $P_{X, Y}=\frac{u}{N+1}$ (particle hops right) and $P_{Y, X}=\frac{q}{N+1}$ (particle hops left).

- If $X=\circ B$ and $Y=\bullet B$ then $P_{X, Y}=\frac{\alpha}{N+1}$ (particle enters from the left).

- If $X=B \bullet$ and $Y=B \circ$ then $P_{X, Y}=\frac{\beta}{N+1}$ (particle exits to the right).

- If $X=\bullet B$ and $Y=\circ B$ then $P_{X, Y}=\frac{\gamma}{N+1}$ (particle exits to the left).

- If $X=B \circ$ and $Y=B \bullet$ then $P_{X, Y}=\frac{\delta}{N+1}$ (particle enters from the right).

- Otherwise $P_{X, Y}=0$ for $Y \neq X$ and $P_{X, X}=1-\sum_{X \neq Y} P_{X, Y}$.

See Figure 1 for an illustration of the four states, with transition probabilities, for the case $N=2$. The probabilities on the loops are determined by the fact that the sum of the probabilities on all outgoing arrows from a given state must be 1 .

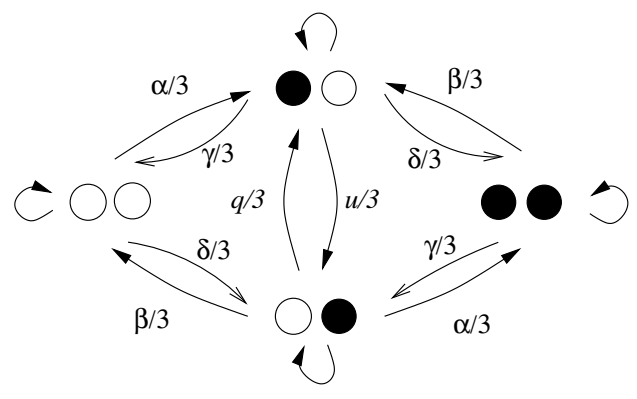

Figure 1. The state diagram of the ASEP for $N=2$

In the long time limit, the system reaches a steady state where all the probabilities $P_{n}\left(\tau_{1}, \tau_{2}, \ldots, \tau_{N}\right)$ of finding the system in configurations $\left(\tau_{1}, \tau_{2}, \ldots, \tau_{N}\right)$ are stationary. More specifically, the stationary distribution is the unique (up to scaling) eigenvector of the transition matrix of the Markov chain with eigenvalue 1.

3.2. The two-species exclusion process. The two-species ASEP is a generalization of the ASEP which involves two kinds of particles, heavy and light. We will denote a heavy particle by a 2 and a light particle by a 1 . We will also denote a hole (or the absence of a particle) by a 0 . In the two-species ASEP, heavy particles 
behave exactly as do particles in the usual ASEP: they can hop in and out of the boundary, and they can hop left and right in the lattice (swapping places with a hole or with a light particle). Light particles cannot hop in and out of the boundary, but they may hop left and right in the lattice (swapping places with a hole or with a heavy particle). Therefore the number $r$ of light particles is conserved.

Definition 3.2. Let $\alpha, \beta, \gamma, \delta, q$, and $u$ be constants such that $0 \leq \alpha \leq 1,0 \leq \beta \leq 1$, $0 \leq \gamma \leq 1,0 \leq \delta \leq 1,0 \leq q \leq 1$, and $0 \leq u \leq 1$. Let $B_{N, r}$ be the set of all words in $\{0,1,2\}^{N}$ which contain precisely $r 1$ 's; note that $\left|B_{N, r}\right|=\left(\begin{array}{c}N \\ r\end{array}\right) 2^{N-r}$. The two-species ASEP is the Markov chain on $B_{N, r}$ with transition probabilities:

- If $X=A 21 B$ and $Y=A 12 B$, or if $X=A 20 B$ and $Y=A 02 B$, or if $X=A 10 B$ and $Y=A 01 B$, then $P_{X, Y}=\frac{u}{N+1}$ and $P_{Y, X}=\frac{q}{N+1}$.

- If $X=0 B$ and $Y=2 B$ then $P_{X, Y}=\frac{\alpha}{N+1}$.

- If $X=B 2$ and $Y=B 0$ then $P_{X, Y}=\frac{\beta}{N+1}$.

- If $X=2 B$ and $Y=0 B$ then $P_{X, Y}=\frac{\gamma}{N+1}$.

- If $X=B 0$ and $Y=B 2$ then $P_{X, Y}=\frac{\delta}{N+1}$.

- Otherwise $P_{X, Y}=0$ for $Y \neq X$ and $P_{X, X}=1-\sum_{X \neq Y} P_{X, Y}$.

Note that if $r=0$, i.e. there are no light particles, then the two-species ASEP is simply the usual ASEP.

3.3. The Matrix Ansatz. We now explain the Matrix Ansatz, a technique introduced in [DEHP93] for computing the stationary distribution of the ASEP. We also present Uchiyama's generalization of the Matrix Ansatz [Uch08 to the two-species case. Finally we will recall the solution to the Matrix Ansatz which was found in USW04.

For convenience, we now set $u=1$. Derrida, Evans, Hakim, and Pasquier [DEHP93] proved the following theorem.

Theorem 3.3. DEHP93] Suppose that there are matrices $D$ and $E$, and vectors $\langle W|$ and $|V\rangle$ such that the following relations hold:

- $\langle W|(\alpha E-\gamma D)=\langle W|$

- $(\beta D-\delta E)|V\rangle=|V\rangle$

- $D E-q E D=D+E$

Let $Z_{N}=\left\langle W\left|(D+E)^{N}\right| V\right\rangle$. Then in the ASEP on a lattice of $N$ sites, the steady state probability of state $\left(\tau_{1}, \ldots, \tau_{N}\right)$ is equal to

$$
\frac{\left\langle W\left|\prod_{i=1}^{N}\left[\mathbb{1}\left(\tau_{i}=\bullet\right) D+\mathbb{1}\left(\tau_{i}=\circ\right) E\right]\right| V\right\rangle}{Z_{N}} .
$$

For example, the steady state probability of state $(\bullet, \circ, \circ, \bullet, \bullet)$ is equal to $\frac{\langle W|D E E D D| V\rangle}{Z_{5}}$.

Uchiyama generalized the Matrix Ansatz to the setting of the two-species exclusion process.

Theorem 3.4. [Uch08] Suppose that there are matrices D, E, A, and vectors $\langle W|$ and $|V\rangle$ such that the following relations hold: 
- $\langle W|(\alpha E-\gamma D)=\langle W|$

- $(\beta D-\delta E)|V\rangle=|V\rangle$

- $D E-q E D=D+E$

- $D A=q A D+A$

- $A E=q E A+A$.

Then in the two-species ASEP on a lattice of $N$ sites with precisely $r$ light particles, the steady state probability of state $\left(\tau_{1}, \ldots, \tau_{n}\right)$ is equal to

$$
\frac{\left\langle W\left|\prod_{i=1}^{N}\left[\mathbb{1}\left(\tau_{i}=2\right) D+\mathbb{1}\left(\tau_{i}=1\right) A+\mathbb{1}\left(\tau_{i}=0\right) E\right]\right| V\right\rangle}{\left[y^{r}\right]\left\langle W\left|(D+E+y A)^{N}\right| V\right\rangle}
$$

For example, the steady state probability of state $(2,0,1,1,2,2)$ is equal to $\frac{\langle W|D E A A D D| V\rangle}{Z_{6,2}}$.

3.4. A solution to the Matrix Ansatz. Now consider the following tridiagonal matrices, which were introduced by Uchiyama, Sasamoto and Wadati in [USW04].1]

$$
\begin{aligned}
& \mathrm{d}=\left[\begin{array}{cccc}
d_{0}^{\natural} & d_{0}^{\sharp} & 0 & \cdots \\
d_{0}^{b} & d_{1}^{\natural} & d_{1}^{\sharp} & \\
0 & d_{1}^{b} & d_{2}^{\natural} & \ddots \\
\vdots & & \ddots & \ddots
\end{array}\right], \quad \mathrm{e}=\left[\begin{array}{cccc}
e_{0}^{\natural} & e_{0}^{\sharp} & 0 & \cdots \\
e_{0}^{b} & e_{1}^{\natural} & e_{1}^{\sharp} & \\
0 & e_{1}^{b} & e_{2}^{\natural} & \ddots \\
\vdots & & \ddots & \ddots
\end{array}\right] \text {, where } \\
& d_{n}^{\natural}:=d_{n}^{\natural}(a, b, c, d)=\frac{q^{n-1}}{\left(1-q^{2 n-2} a b c d\right)\left(1-q^{2 n} a b c d\right)} \\
& \times\left[b d(a+c)+(b+d) q-a b c d(b+d) q^{n-1}-\{b d(a+c)+a b c d(b+d)\} q^{n}\right. \\
& \left.-b d(a+c) q^{n+1}+a b^{2} c d^{2}(a+c) q^{2 n-1}+a b c d(b+d) q^{2 n}\right] \text {, } \\
& e_{n}^{\natural}:=e_{n}^{\natural}(a, b, c, d)=\frac{q^{n-1}}{\left(1-q^{2 n-2} a b c d\right)\left(1-q^{2 n} a b c d\right)} \\
& \times\left[a c(b+d)+(a+c) q-a b c d(a+c) q^{n-1}-\{a c(b+d)+a b c d(a+c)\} q^{n}\right. \\
& \left.-a c(b+d) q^{n+1}+a^{2} b c^{2} d(b+d) q^{2 n-1}+a b c d(a+c) q^{2 n}\right] \text {, } \\
& d_{n}^{\sharp}:=d_{n}^{\sharp}(a, b, c, d)=1, \quad \quad d_{n}^{b}:=d_{n}^{b}(a, b, c, d)=-\frac{q^{n} b d}{\left(1-q^{n} a c\right)\left(1-q^{n} b d\right)} \mathcal{A}_{n} \\
& e_{n}^{\sharp}:=e_{n}^{\sharp}(a, b, c, d)=-q^{n} a c, \quad e_{n}^{b}:=e_{n}^{b}(a, b, c, d)=\frac{1}{\left(1-q^{n} a c\right)\left(1-q^{n} b d\right)} \mathcal{A}_{n}, \text { and } \\
& \mathcal{A}_{n}:=\mathcal{A}_{n}(a, b, c, d) \\
& =\frac{\left(1-q^{n-1} a b c d\right)\left(1-q^{n+1}\right)\left(1-q^{n} a b\right)\left(1-q^{n} a c\right)\left(1-q^{n} a d\right)\left(1-q^{n} b c\right)\left(1-q^{n} b d\right)\left(1-q^{n} c d\right)}{\left(1-q^{2 n-1} a b c d\right)\left(1-q^{2 n} a b c d\right)^{2}\left(1-q^{2 n+1} a b c d\right)} .
\end{aligned}
$$

\footnotetext{
${ }^{1}$ Actually we have slightly modified the definitions of $d_{i}^{\sharp}, d_{i}^{b}, e_{i}^{\sharp}$, and $e_{i}^{b}$ which appeared in [USW04] but it is easy to check that both the matrices we are using here and the original matrices of [USW04] satisfy Lemma 3.6 .
} 
Remark 3.5. These matrices have the property that the coefficients in the nth row of $\mathrm{d}+\mathrm{e}$ are the coefficients in the three-term recurrence for the Askey-Wilson polynomials.

Lemma 3.6 below uses the following change of variables between $a, b, c, d$ and $\alpha, \beta, \gamma, \delta$.

$$
\begin{aligned}
& a=\frac{1-q-\alpha+\gamma+\sqrt{(1-q-\alpha+\gamma)^{2}+4 \alpha \gamma}}{2 \alpha} \\
& c=\frac{1-q-\alpha+\gamma-\sqrt{(1-q-\alpha+\gamma)^{2}+4 \alpha \gamma}}{2 \alpha} \\
& b=\frac{1-q-\beta+\delta+\sqrt{(1-q-\beta+\delta)^{2}+4 \beta \delta}}{2 \beta} \\
& d=\frac{1-q-\beta+\delta-\sqrt{(1-q-\beta+\delta)^{2}+4 \beta \delta}}{2 \beta} .
\end{aligned}
$$

Note that this change of variables can be inverted via

$$
\begin{array}{lll}
\alpha=\frac{1-q}{1+a c+a+c}, & \beta & =\frac{1-q}{1+b d+b+d}, \\
\gamma=\frac{-(1-q) a c}{1+a c+a+c}, & \delta & =\frac{-(1-q) b d}{1+b d+b+d} .
\end{array}
$$

Lemma 3.6. USW04 Let $D=\frac{1}{1-q}(\mathbb{1}+\mathrm{d})$ and $E=\frac{1}{1-q}(\mathbb{1}+\mathrm{e})$, where $\mathbb{1}$ is the identity matrix. Also, use the equations (4) through (77) to express $D$ and $E$ in terms of $\alpha, \beta, \gamma, \delta$, and $q$. Let $\langle W|=(1,0,0, \ldots)$ and $|V\rangle=\left\langle\left. W\right|^{T}\right.$. Then $D, E,\langle W|$, and $|V\rangle$ give a solution to the Matrix Ansatz of Theorem 3.3.

Moreover, Uchiyama [uch08] observed that if $A:=D E-E D$, and $D$ and $E$ satisfy $D E-q E D=D+E$, then it follows that $D A=q A D+A$ and $A E=q E A+A$. Therefore we have the following.

Corollary 3.7. Let $D, E,\langle W|$, and $|V\rangle$ be as in Lemma 3.6, and set $A:=D E-E D$. Then D, E, A, $\langle W|$, and $|V\rangle$ give a solution to the Matrix Ansatz of Theorem 3.4.

In the rest of the paper, we will use the solution of the matrix ansatz in Corollary 3.7, which in turn fixes our definition of the following versions of the partition function. (Note that one gets a definition of the partition function from every solution to the Matrix Ansatz; using a different solution will simply rescale the partition function.)

Definition 3.8. Let $Z_{N}(\xi)=\left\langle W\left|(\xi D+E)^{N}\right| V\right\rangle$ be the fugacity partition function for the ASEP where D,E, $\langle W|$, and $|V\rangle$ are as in Lemma 3.6. We also let $Z_{N, r}(\xi)=$ $\left[y^{r}\right] \frac{\left\langle W\left|(\xi D+E+y A)^{N}\right| V\right\rangle}{\left\langle W\left|A^{r}\right| V\right\rangle}$ be the fugacity partition function for the two-species ASEP. Finally we set $Z_{N}=Z_{N}(1)$ and $Z_{N, r}=Z_{N, r}(1)$ and refer to these quantities as partition functions. 


\section{KoORnWinder moments And partial Motzkin paths}

We start by reviewing the connection between the partition function $Z_{N}$ of the ASEP and moments of Askey-Wilson polynomials. We will then explain how to interpret $Z_{N}$ as a generating function for weighted Motzkin paths. Finally we will use the celebrated lemma of Karlin-McGregor-Lindström-Gessel-Viennot [KM59b, KM59a, Lin73, GV85] on determinants and non-intersecting lattice paths to express Koornwinder moments in terms of partial weighted Motzkin paths.

4.1. The partition function of the ASEP, Askey-Wilson moments, and Koornwinder moments. Using results from [USW04, we proved in [CSSW12. that there is a close relationship between the fugacity partition function $Z_{N}$ of the ASEP and Askey-Wilson moments $\mu_{N}$.

Theorem 4.1. CSSW12, Theorem 1.11] Recall that $Z_{N}(\xi)=Z_{N}(\xi ; \alpha, \beta, \gamma, \delta ; q)=$ $\left\langle W\left|(\xi D+E)^{N}\right| V\right\rangle$ be the fugacity partition function of the ASEP, where $D, E,\langle W|$, and $|V\rangle$ are as in Lemma 3.6. Then the $N^{\text {th }}$ Askey-Wilson moment is equal to

$$
\mu_{N}(a, b, c, d \mid q)=\frac{(1-q)^{N}}{2^{N} i^{N}} Z_{N}(-1 ; \alpha, \beta, \gamma, \delta ; q), 2
$$

where $i^{2}=-1$ and

$$
\alpha=\frac{1-q}{1-a c+a i+c i}, \beta=\frac{1-q}{1-b d-b i-d i}, \gamma=\frac{(1-q) a c}{1-a c+a i+c i}, \delta=\frac{(1-q) b d}{1-b d-b i-d i} .
$$

Recall from Lemma 2.7 our determinantal expression for Koornwinder moments. Because of the close relationship between the partition function of the ASEP and Askey-Wilson moments (see Theorem 4.1), we define another version of Koornwinder moments as follows.

Definition 4.2. Given a partition $\lambda=\left(\lambda_{1}, \lambda_{2}, \ldots, \lambda_{m}\right)$, we define the Koornwinder moment at $q=t$ to be

$$
K_{\lambda}(\xi)=K_{\lambda}(\xi ; \alpha, \beta, \gamma, \delta ; q)=\frac{\operatorname{det}\left(Z_{\lambda_{i}+m-i+m-j}(\xi)\right)_{i, j=1}^{m}}{\operatorname{det}\left(Z_{2 m-i-j}(\xi)\right)_{i, j=1}^{m}},
$$

where $Z_{N}$ is as in Definition 3.8 .

The fact that we refer to the quantities $K_{\lambda}(\xi)$ as Koornwinder moments is justified by the following result.

Proposition 4.3. Let $\lambda=\left(\lambda_{1}, \ldots, \lambda_{m}\right)$ be a partition. We have that

$$
M_{\lambda}(a, b, c, d \mid q)=\left(\frac{1-q}{2 i}\right)^{|\lambda|} K_{\lambda}(-1 ; \alpha, \beta, \gamma, \delta ; q),
$$

where $|\lambda|=\sum_{i} \lambda_{i}$, and $\alpha, \beta, \gamma, \delta$ are related to $a, b, c, d$ as in (10).

\footnotetext{
${ }^{2}$ The formula we give here does not include the factor $\prod_{j=0}^{N-1}\left(\alpha \beta-\gamma \delta q^{j}\right)$, because we are working here with the partition function coming from the $D$ and $E$ from Lemma 3.6. rather than the rescaled version which was used in CSSW12.
} 
Proof. Let $\theta=\frac{1-q}{2 i}$. So $Z_{N}(-1 ; \alpha, \beta, \gamma, \delta ; q)=\theta^{-N} \mu_{N}(a, b, c, d \mid q)$. Then we have that

$$
\begin{aligned}
K_{\lambda}(-1) & =\frac{\operatorname{det}\left(Z_{\lambda_{i}+m-i+m-j}(-1)\right)_{i, j=1}^{m}}{\operatorname{det}\left(Z_{2 m-i-j}(-1)\right)_{i, j=1}^{m}} \\
& =\frac{\operatorname{det}\left(\theta^{-\left(\lambda_{i}+m-i+m-j\right)} \mu_{\lambda_{i}+m-i+m-j}\right)_{i, j=1}^{m}}{\operatorname{det}\left(\theta^{-(2 m-i-j)} \mu_{2 m-i-j}\right)_{i, j=1}^{m}} \\
& =\frac{\theta^{-\left(\lambda_{1}+\cdots+\lambda_{m}+m(m-1)\right)} \operatorname{det}\left(\mu_{\lambda_{i}+m-i+m-j}\right)_{i, j=1}^{m}}{\theta^{-m(m-1)} \operatorname{det}\left(\mu_{2 m-i-j}\right)_{i, j=1}^{m}} \\
& =\theta^{-\left(\lambda_{1}+\cdots+\lambda_{m}\right)} M_{\lambda} .
\end{aligned}
$$

From now on, when we refer to Koornwinder moments, we will be referring to Definition 4.2 .

Because of its probabilistic interpretation, the quantity $Z_{N}$ must be positive whenever we specialize the parameters $\alpha, \beta, \gamma, \delta$, and $q$ to be positive numbers between 0 and 1. Moreover, we proved in [CW11, CW13] that (up to a normalizing factor) $Z_{N}$ is a polynomial in $\alpha, \beta, \gamma, \delta, q$ with positive coefficients; it can be expressed as a sum over staircase tableaux. This prompted Rains to conjecture the following.

Conjecture 4.4. The Koornwinder moment $K_{\lambda}(\xi)$ is a polynomial in $\alpha, \beta, \gamma, \delta, q, \xi$ with positive coefficients (up to a normalizing factor).

Since Theorem 1.1 implies that $K_{(N-r, 0,0, \ldots, 0)}(\xi)$ is proportional to the fugacity partition function $Z_{N, r}(\xi)$ for the two-species ASEP, it follows that the Koornwinder moment $K_{(N-r, 0,0, \ldots, 0)}$ is positive when we specialize the parameters $\alpha, \beta, \gamma, \delta, q$ to be positive numbers between 0 and 1. Moreover, in [CMW17], we will give a tableaux interpretation of $Z_{N, r}$, which implies that $Z_{N, r}(\xi)$ is a polynomial in $\xi, \alpha, \beta, \gamma, \delta$, and $q$, with positive coefficients. Finally, see Section 8 for some evidence towards Conjecture 4.4 when $q=\xi=1$.

4.2. Weighted Motzkin paths and the partition function of the ASEP. Recall that a Motzkin path of length $N$ is a path in the $x y$ plane from $(0,0)$ to $(N, 0)$ which consists of steps northeast $(1,1)$, east $(1,0)$, and southeast $(1,-1)$, and never dips below the $x$-axis. One often associates a weight to each of the three kinds of steps, based on the height at which the step begins. The weight of a given Motzkin path is then the product of the weights of all of its steps, and such a path is called a weighted Motzkin path. If $\mathcal{C}=\left(c_{i j}\right)$ is a tridiagonal matrix rows and columns indexed by the non-negative integers, then we can use each nonzero entry $c_{i j}$ to weight a step in a Motzkin path that starts at height $i$ and ends at height $j$. We call such a Motzkin path a $\mathcal{C}$-Motzkin path, though we will sometimes drop the $\mathcal{C}$ if it is understood.

The following lemma is obvious.

Lemma 4.5. Let $\mathcal{C}$ be a tridiagonal matrix as above, and let $\langle W|=(1,0,0, \ldots)$ and $|V\rangle=\left\langle\left. W\right|^{T}\right.$. Let $\mathcal{Z}_{N}=\left\langle W\left|\mathcal{C}^{N}\right| V\right\rangle$. Then $\mathcal{Z}_{N}$ is the generating function for all 
$\mathcal{C}$-Motzkin paths of length $N$, i.e. it is the sum of the weights of all $\mathcal{C}$-Motkin paths from $(0,0)$ to $(N, 0)$.

We will now give a general result relating a certain ratio of determinants to partial Motzkin paths, which we will use to subsequently relate Koornwinder moments to partial Motzkin paths. Note that it is well-known that there is a link between (partial) Motzkin paths and moments of (one-variable) orthogonal polynomials, see for example [Vie, Chapter 1, Proposition 17] and also [Vie85]. However, our treatment here will be self-contained.

We say a partial Motzkin path is a path in the $x y$ plane from $(0,0)$ to $(N, r)$ which consists of steps northeast $(1,1)$, east $(1,0)$, and southeast $(1,-1)$, and never dips below the $x$-axis. As before, we can use entries $c_{i j}$ of a tridiagonal matrix $\mathcal{C}$ to associate the weight $c_{i j}$ to a step which starts at height $i$ and ends at height $j$. The weight of a partial Motzkin path is then the product of the weights of all of its steps. We have the following result.

We define the following ratio of determinants:

$$
\mathcal{K}_{\left(\lambda_{1}, \ldots, \lambda_{n}\right)}=\frac{\operatorname{det}\left|\begin{array}{cccc}
\mathcal{Z}_{\lambda_{1}+2 n-2} & \mathcal{Z}_{\lambda_{1}+2 n-3} & \ldots & \mathcal{Z}_{\lambda_{1}+n-1} \\
\mathcal{Z}_{\lambda_{2}+2 n-3} & \mathcal{Z}_{\lambda_{2}+2 n-4} & \ldots & \mathcal{Z}_{\lambda_{2}+n-2} \\
\vdots & \vdots & & \vdots \\
\mathcal{Z}_{\lambda_{n}+n-1} & \mathcal{Z}_{\lambda_{n}+n-2} & \ldots & \mathcal{Z}_{\lambda_{n}}
\end{array}\right|}{\operatorname{det}\left|\begin{array}{cccc}
\mathcal{Z}_{2 n-2} & \mathcal{Z}_{2 n-3} & \ldots & \mathcal{Z}_{n-1} \\
\mathcal{Z}_{2 n-3} & \mathcal{Z}_{2 n-4} & \ldots & \mathcal{Z}_{n-2} \\
\vdots & \vdots & & \vdots \\
\mathcal{Z}_{n-1} & \mathcal{Z}_{n-2} & \ldots & \mathcal{Z}_{0}
\end{array}\right|}
$$

Theorem 4.6. Let $\mathcal{C}$ be a tridiagonal matrix, and let $\mathcal{C} \operatorname{Motz}(N, r)$ be the generating function for all $\mathcal{C}$ - partial Motzkin paths which start at $(0,0)$ and end at $(N, r)$. Let $\langle W|=(1,0,0, \ldots)| V\rangle=,\left\langle\left. W\right|^{T}\right.$, and $\left.\mid V^{r}\right\rangle=(0, \ldots, 0,1,0,0, \ldots)^{T}$, where the 1 is in the rth position (and coordinates are indexed by the non-negative integers). Let $\mathcal{Z}_{N}=\left\langle W\left|\mathcal{C}^{N}\right| V\right\rangle$ and $k_{r}=\prod_{i=0}^{r-1} c_{i, i+1}$. Then we have that

$$
\frac{1}{k_{r}} \mathcal{C} \operatorname{Motz}(N, r)=\frac{1}{k_{r}}\left\langle W\left|\mathcal{C}^{N}\right| V^{r}\right\rangle=\mathcal{K}_{(N-r, 0,0, \ldots, 0)},
$$

where there are precisely $r$ 's in $(N-r, 0,0, \ldots, 0)$. 
Proof. The leftmost equality in (13) is obvious. To relate these quantities to the ratio of determinants

$$
\mathcal{K}_{(N-r, 0,0, \ldots, 0)}=\frac{\operatorname{det}\left|\begin{array}{cccc}
\mathcal{Z}_{N+r} & \mathcal{Z}_{N+r-1} & \ldots & \mathcal{Z}_{N} \\
\mathcal{Z}_{2 r-1} & \mathcal{Z}_{2 r-2} & \ldots & \mathcal{Z}_{r-1} \\
\vdots & \vdots & & \vdots \\
\mathcal{Z}_{r} & \mathcal{Z}_{r-1} & \ldots & \mathcal{Z}_{0}
\end{array}\right|}{\operatorname{det}\left|\begin{array}{cccc}
\mathcal{Z}_{2 r} & \mathcal{Z}_{2 r-1} & \ldots & \mathcal{Z}_{r} \\
\mathcal{Z}_{2 r-1} & \mathcal{Z}_{2 r-2} & \ldots & \mathcal{Z}_{r-1} \\
\vdots & \vdots & & \vdots \\
\mathcal{Z}_{r} & \mathcal{Z}_{r-1} & \ldots & \mathcal{Z}_{0}
\end{array}\right|}
$$

at the right, we now use the well-known Karlin-McGregor-Lindström-Gessel-Viennot Lemma [KM59b, KM59a, Lin73, GV85] (which we will henceforth refer to as the KMLGV Lemma). This lemma will give a combinatorial interpretation of both the numerator and denominator.
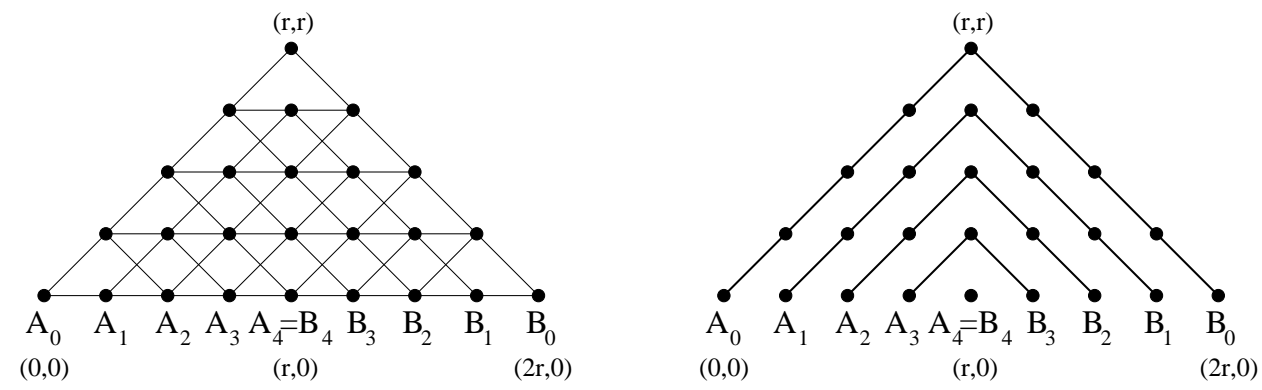

Figure 2. An acyclic directed graph (we consider all edges to be directed either east, northeast, or southeast) corresponding to the denominator of (13) when $r=4$, and the unique collection of pairwise vertex-disjoint paths from $\left\{A_{0}, \ldots, A_{r}\right\}$ to $\left\{B_{0}, \ldots, B_{r}\right\}$ in this graph.

Consider the acyclic directed graph shown at the left of Figure 2 (for the case that $r=4)$. Although we haven't shown the orientations of the edges, we consider all edges to be directed either east, northeast, or southeast. The points $A_{0}, A_{1}, \ldots$, $A_{r}=B_{r}, B_{r-1}, B_{r-2}, \ldots, B_{0}$ are at the lattice points $(0,0),(1,0), \ldots,(r, 0),(r+$ $1,0),(r+2,0), \ldots,(2 r, 0)$, respectively. We weight each edge of the graph which goes from height $i$ to height $j$ by the entry $c_{i j}$ of $\mathcal{C}$. Then we define an $(r+1) \times(r+1)$ weight matrix $M^{\text {den }}=\left(M_{i j}^{\text {den }}\right)$ as follows. Its rows and columns are indexed by $\{0,1, \ldots, r\}$, and $M_{i j}^{\text {den }}$ is defined to be the weights of all paths in the graph from $A_{i}$ to $B_{j}$. In other words, $M_{i j}^{\text {den }}$ is the generating function for all $\mathcal{C}$-Motzkin paths of length $2 r-i-j$. Applying Lemma 4.5, we have that $M_{i j}^{\text {den }}=\mathcal{Z}_{2 r-i-j}$, and hence $M^{\text {den }}$ is the matrix appearing in the denominator of (13).

But now by the KMLGV Lemma, the determinant $\operatorname{det} M^{\text {den }}$ is the generating function for pairwise vertex-disjoint path collections from $\left\{A_{0}, \ldots, A_{r}\right\}$ to $\left\{B_{0}, \ldots, B_{r}\right\}$. It is easy to see that there is only one such path collection, which is shown at the 
right of Figure 2, Note that [Kra99, Theorem 11] can also be used to give a formula for this Hankel determinant.
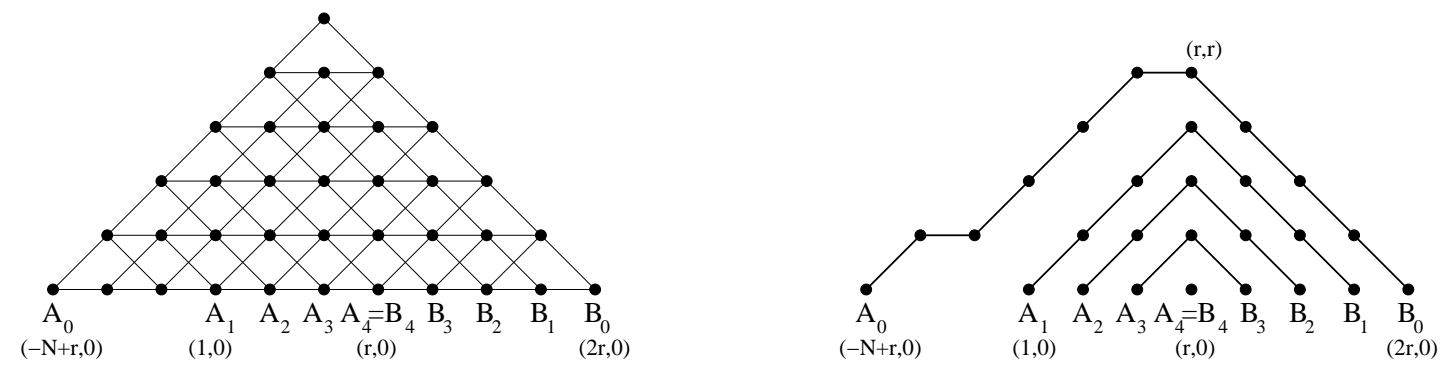

FiguRE 3. An acyclic directed graph (we consider all edges to be directed either east, northeast, or southeast) corresponding to the numerator of (13) when $r=4$. Now there are multiple collections of pairwise vertex-disjoint paths from $\left\{A_{0}, \ldots, A_{r}\right\}$ to $\left\{B_{0}, \ldots, B_{r}\right\}$, but all of them use the set of paths from $A_{i}$ to $B_{i}$ for $1 \leq i \leq 4$ shown at the right of the figure.

Now consider the acyclic directed graph shown at the left of Figure 3 (for the case that $r=4$ ). Again we consider all edges to be directed either east, northeast, or southeast. The points $A_{0}, A_{1}, \ldots, A_{r}=B_{r}, B_{r-1}, B_{r-2}, \ldots, B_{0}$ are at the lattice points $(-N+r, 0),(1,0), \ldots,(r, 0),(r+1,0),(r+2,0), \ldots,(2 r, 0)$, respectively. We again weight edges of the graph using entries of $\mathcal{C}$. Then we define the $(r+1) \times(r+1)$ matrix $M^{\text {num }}=\left(M_{i j}^{\text {num }}\right)$, with rows and columns indexed by $\{0,1, \ldots, r\}$, as follows. $M_{i j}^{\text {num }}$ is defined to be the weights of all Motzkin paths from $A_{i}$ to $B_{j}$. Using Lemma 4.5, it follows that $M^{\text {num }}$ is the matrix appearing in the numerator of (13).

By the KMLGV Lemma, the determinant $\operatorname{det} M^{\text {num }}$ is the generating function for pairwise vertex-disjoint path collections from $\left\{A_{0}, \ldots, A_{r}\right\}$ to $\left\{B_{0}, \ldots, B_{r}\right\}$. It is easy to see that such path collections must consist of the paths from $A_{i}$ to $B_{i}$ for $1 \leq i \leq r$ shown at the right of Figure 3 , together with an arbitrary Motzkin path from $A_{0}$ to $B_{0}$ which goes through the point $(r, r)$ and whose last $r$ steps are southeast.

We now have combinatorial interpretations for both $\operatorname{det} M^{\text {num }}$ and $\operatorname{det} M^{\text {den }}$. Comparing them, we see that the ratio $\frac{\operatorname{det} M^{\text {num }}}{\operatorname{det} M^{\operatorname{den}}}$ is equal to the generating function for the partial Motzkin paths from $(-N+r, 0)$ to $(r, r)$ divided by the weight of the unique partial Motzkin path from $(0,0)$ to $(r, r)$. Clearly the generating function for the partial Motzkin paths from $(-N+r, 0)$ to $(r, r)$ equals the generating function for the partial Motzkin paths from $(0,0)$ to $(N, r)$. And the weights of the up steps in our Motzkin paths are $c_{i, i+1}$, so the weight of that unique partial Motzkin path is $c_{0,1} c_{1,2} \ldots c_{r-1, r}$. This completes the proof.

Corollary 4.7. Now we choose the tridiagonal matrix $C=\xi D+E$, where $D$ and $E$ are as in Lemma 3.6. Let $C \operatorname{Motz}(N, r)$ be the generating function for all $C$-partial 
Motzkin paths which start at $(0,0)$ and end at $(N, r)$. Then we have that

$$
K_{(N-r, 0,0, \ldots, 0)}=\frac{1}{k_{r}}\left\langle W\left|C^{N}\right| V^{r}\right\rangle=\frac{1}{k_{r}} C \operatorname{Motz}(N, r),
$$

where there are precisely $r$ 0's in $(N-r, 0,0, \ldots, 0)$, and $k_{r}=\frac{\prod_{i=0}^{r-1}\left(\xi-q^{i} a c\right)}{(1-q)^{r}}$.

To prove Theorem 1.1, we now need to relate $\left\langle W\left|(\xi D+E)^{N}\right| V^{r}\right\rangle$ to the fugacity partition function for the 2-species ASEP.

\section{A Jacobi-Trudi formula for general KoORnWinder moments}

In this section we will prove a general Jacobi-Trudi type result in Theorem 5.1 , which expresses the quantity $\mathcal{K}_{\lambda}$ (see (12)) in terms of the quantities $\mathcal{K}_{(m, 0, \ldots, 0)}$. Our proof techniques build on those used in the proof of Theorem 4.7. We again let $\mathcal{C}=\left(c_{i j}\right)$ be a tridiagonal matrix. Edges of graphs that we will construct here are all directed northeast, east, or southeast, and each edge from height $i$ to $j$ is weighted by $c_{i j}$.

Theorem 5.1. Let $\lambda=\left(\lambda_{1}, \ldots, \lambda_{n}\right)$ be a partition. Then

$$
\mathcal{K}_{\lambda}=\operatorname{det}\left(\mathcal{K}_{\left(\lambda_{i}+j-i, 0,0, \ldots, 0\right)}\right)_{i, j=1}^{n},
$$

where the term on the right-hand-side has precisely $n-j 0$ 's.

Note that Theorem 5.1 immediately implies Corollary 5.2, which expresses a general Koornwinder moment $K_{\lambda}(\xi)$ in terms of the "complete homogeneous" Koornwinder moments $K_{(m, 0, \ldots, 0)}(\xi)$.

Corollary 5.2. Let $\lambda=\left(\lambda_{1}, \ldots, \lambda_{n}\right)$ be a partition. Then

$$
K_{\lambda}(\xi)=\operatorname{det}\left(K_{\left(\lambda_{i}+j-i, 0,0, \ldots, 0\right)}(\xi)\right)_{i, j=1}^{n},
$$

where the term on the right-hand-side has precisely $n-j 0$ 's.

We now prove Theorem 5.1

Proof. We start by analyzing the left-hand side of (15). By definition, we have that

$$
\mathcal{K}_{\left(\lambda_{1}, \ldots, \lambda_{n}\right)}=\frac{\operatorname{det}\left|\begin{array}{cccc}
\mathcal{Z}_{\lambda_{1}+2 n-2} & \mathcal{Z}_{\lambda_{1}+2 n-3} & \ldots & \mathcal{Z}_{\lambda_{1}+n-1} \\
\mathcal{Z}_{\lambda_{2}+2 n-3} & \mathcal{Z}_{\lambda_{2}+2 n-4} & \ldots & \mathcal{Z}_{\lambda_{2}+n-2} \\
\vdots & \vdots & & \vdots \\
\mathcal{Z}_{\lambda_{n}+n-1} & \mathcal{Z}_{\lambda_{n}+n-2} & \ldots & \mathcal{Z}_{\lambda_{n}}
\end{array}\right|}{\operatorname{det}\left|\begin{array}{cccc}
\mathcal{Z}_{2 n-2} & \mathcal{Z}_{2 n-3} & \ldots & \mathcal{Z}_{n-1} \\
\mathcal{Z}_{2 n-3} & \mathcal{Z}_{2 n-4} & \ldots & \mathcal{Z}_{n-2} \\
\vdots & \vdots & & \vdots \\
\mathcal{Z}_{n-1} & \mathcal{Z}_{n-2} & \ldots & \mathcal{Z}_{0}
\end{array}\right|}
$$

Using the same arguments as in the proof of Theorem 4.7, we interpret the matrix in the numerator of (17) as the weight matrix associated to the acyclic directed graph at the left of Figure 4, where edges from height $i$ to height $j$ are weighted by $c_{i j}$. Note 

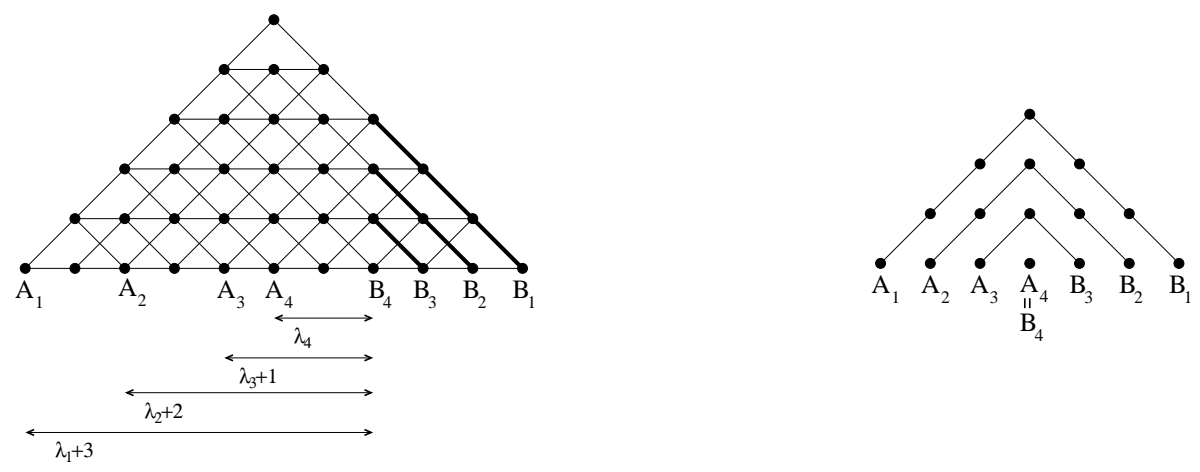

FiguRE 4. An acyclic directed graph (all edges are directed either east, northeast, or southeast), whose weight matrix is given by (17), when $n=4$.

that the points $A_{1}, \ldots, A_{n}$, and $B_{n}, \ldots, B_{1}$ all lie on the $x$-axis, with the $B_{i}$ 's at unit distance apart. The positions of the $A_{i}$ 's have been chosen so that the distance between $A_{i}$ and $B_{n}$ is $\lambda_{i}+n-i$. By Lemma 4.5, the $i j$ entry $\mathcal{Z}_{\lambda_{i}+2 n-i-j}$ of the matrix in the numerator of (17) is equal to the generating function for Motzkin paths from $A_{i}$ to $B_{j}$, hence this matrix is the weight matrix associated to the graph at the left of Figure 4, By the KMLGV Lemma, its determinant is the generating function for pairwise vertex-disjoint path collections from $\left\{A_{1}, \ldots, A_{r}\right\}$ to $\left\{B_{1}, \ldots, B_{r}\right\}$. Note that all such path collections must use the southeast edges which are shown in bold at the left of Figure 4. Moreover, as we showed in the proof of Theorem 4.7, the determinant in the denominator of (17) is equal to the weight of the path collection shown at the right of Figure 4. Therefore the ratio of the determinants is equal to the generating function for pairwise-disjoint non-intersecting paths from $A_{1}, \ldots, A_{n}$ to the lattice points $B_{1}, \ldots, B_{4}$ in Figure 5, divided by the product of the weights of all the up steps in the path collection shown at the right of Figure 4 . That product is equal to $k_{1} k_{2} \ldots k_{n-1}$, where $k_{r}=\prod_{i=0}^{r-1} c_{i, i+1}$.

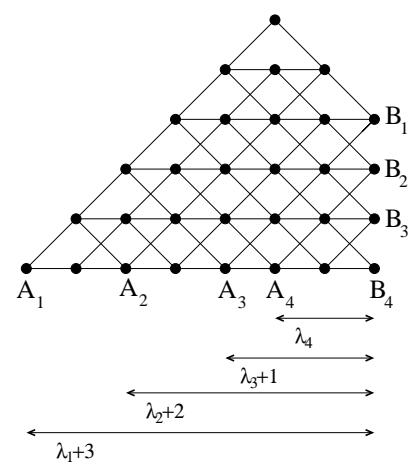

FiguRE 5. An acyclic directed graph, shown for $n=4$. Edges are oriented northeast, east, or southeast. 
We now consider the right-hand side of (15). We have that (18)

$$
\operatorname{det}\left(\mathcal{K}_{\left(\lambda_{i}+j-i, 0,0, \ldots, 0\right)}\right)_{i, j=1}^{n}=\operatorname{det}\left|\begin{array}{cccc}
\mathcal{K}_{\left(\lambda_{1}, 0, \ldots, 0\right)} & \mathcal{K}_{\left(\lambda_{1}+1,0, \ldots, 0\right)} & \ldots & \mathcal{K}_{\left(\lambda_{1}+n-1\right)} \\
\mathcal{K}_{\left(\lambda_{2}-1,0, \ldots, 0\right)} & \mathcal{K}_{\left(\lambda_{2}, 0, \ldots, 0\right)} & \ldots & \mathcal{K}_{\left(\lambda_{2}+n-2\right)} \\
\vdots & \vdots & & \vdots \\
\mathcal{K}_{\left(\lambda_{n}-n+1,0, \ldots, 0\right)} & \mathcal{K}_{\left(\lambda_{n}-n+2,0, \ldots, 0\right)} & \ldots & \mathcal{K}_{\left(\lambda_{n}\right)}
\end{array}\right|
$$

where the partitions in column $j$ of the matrix contain precisely $n-j 0$ 's. Using Corollary 4.7, we have that (18) is equal to the determinant of

$$
\left|\begin{array}{cccc}
\frac{1}{k_{n-1}} \mathcal{C} \operatorname{Motz}\left(\lambda_{1}+n-1, n-1\right) & \frac{1}{k_{n-2}} \mathcal{C} \operatorname{Motz}\left(\lambda_{1}+n-1, n-2\right) & \ldots & \mathcal{C} M \operatorname{Motz}\left(\lambda_{1}+n-1,0\right) \\
\frac{1}{k_{n-1}} \mathcal{C} \operatorname{Motz}\left(\lambda_{2}+n-2, n-1\right) & \frac{1}{k_{n-2}} \mathcal{C} \operatorname{Motz}\left(\lambda_{2}+n-2, n-2\right) & \ldots & \mathcal{C} \operatorname{Motz}\left(\lambda_{2}+n-2,0\right) \\
\vdots & \vdots & & \vdots \\
\frac{1}{k_{n-1}} \mathcal{C} \operatorname{Motz}\left(\lambda_{n}, n-1\right) & \frac{1}{k_{n-2}} \mathcal{C} \operatorname{Motz}\left(\lambda_{n}, n-2\right) & \ldots & \mathcal{C} \operatorname{Motz}\left(\lambda_{n}, 0\right)
\end{array}\right|,
$$

which is equal to

\begin{tabular}{|c|c|c|}
\hline $\begin{array}{l}\mathcal{C} M o t z\left(\lambda_{1}+n-1, n-1\right) \\
\mathcal{C} M o t z\left(\lambda_{2}+n-2, n-1\right)\end{array}$ & $\begin{array}{l}\mathcal{C} M o t z\left(\lambda_{1}+n-1, n-2\right) \\
\mathcal{C} M o t z\left(\lambda_{2}+n-2, n-2\right)\end{array}$ & $\begin{array}{l}\mathcal{C} \operatorname{Motz}\left(\lambda_{1}+n-1,0\right) \\
\mathcal{C} \operatorname{Motz}\left(\lambda_{2}+n-2,0\right)\end{array}$ \\
\hline $\mathcal{C} \operatorname{Mot} z\left(\lambda_{n}, n-1\right)$ & $\mathcal{C} M o t z\left(\lambda_{n}, n-2\right)$ & $\mathcal{C} M o t z\left(\lambda_{n}, 0\right)$ \\
\hline
\end{tabular}

(19)

But now it's clear that the matrix in (19) is the weight matrix for the directed graph shown in Figure 5, so we are done.

\section{From partial Motzkin Paths to the two-SPECIES ASEP}

Our goal in this section is to prove Theorem 6.1, which expresses the fugacity partition function $Z_{N, r}(\xi)$ in terms of partial Motzkin paths. We also use this theorem to give an integral representation and an explicit closed formula for $Z_{N, r}(\xi)$ (the latter was communicated to us by Dennis Stanton).

Theorem 6.1. Define D, E, $\langle W|$, and $|V\rangle$ as in Lemma 3.6, and set $A=D E-E D$. Using the change of variables from (8) and (9), define

$$
\rho_{r}=\frac{(1-q)^{r}}{\prod_{i=0}^{r-1}\left(\xi-q^{i} a c\right)}=\frac{1}{k_{r}}=\frac{\alpha^{r}(1-q)^{r}}{\prod_{i=0}^{r-1}\left(\alpha \xi+q^{i} \gamma\right)}
$$

Then we have that

$$
\left\langle W\left|(\xi D+E)^{N}\right| V^{r}\right\rangle \cdot \rho_{r}=\left[y^{r}\right] \frac{\left\langle W\left|(\xi D+E+y A)^{N}\right| V\right\rangle}{\left\langle W\left|A^{r}\right| V\right\rangle}=Z_{N, r}(\xi) .
$$

Note that $\left\langle W\left|(\xi D+E)^{N}\right| V^{r}\right\rangle$ is the generating function for $\mathcal{C}$-partial Motzkin paths, with $\mathcal{C}=\xi D+E$, so this gives a combinatorial interpretation for the fugacity partition function $Z_{N, r}(\xi)$ of the two-species ASEP. 
Note that our main theorem, Theorem 1.1, is an immediate consequence of Corollary 4.7 and Theorem 6.1.

Before proving Theorem 6.1, we observe that it directly implies an integral representation for the fugacity partition function.

Corollary 6.2. Using the change of variables from (8) and (9), we have the following integral representation for the fugacity partition function:

$$
Z_{N, r}(\xi)=B_{N, r} \int(\sqrt{\xi}+1 / \sqrt{\xi}+x)^{N} w\left(x, a^{\prime}, b^{\prime}, c^{\prime}, d^{\prime} ; q\right) P_{r}\left(x, a^{\prime}, b^{\prime}, c^{\prime}, d^{\prime} ; q\right) \frac{d x}{4 i \pi x}
$$

where $B_{N, r}$ is a simple constant, $a^{\prime}=a \sqrt{\xi}, \quad b^{\prime}=b / \sqrt{\xi}, \quad c^{\prime}=c \sqrt{\xi}, \quad d^{\prime}=d / \sqrt{\xi}$, $w(x, b, c, d ; q)$ is the Askey-Wilson density and $P_{r}(x, a, b, c, d ; q)$ is the $r^{\text {th }}$ AskeyWilson polynomial. See Section 2 for the definitions of the Askey-Wilson density and polynomials.

Proof. Let $\mathcal{C}$ be a tridiagonal matrix, whose rows encode the recurrence relations defining a family of orthogonal polynomials $\left\{P_{m}(x)\right\}$. Let $f$ be the linear functional expressing the orthogonality relation, i.e. $f\left(P_{m}(x) P_{\ell}(x)\right)=0$ unless $m=\ell$. By [Vie85, Proposition 17, Chapter 1], the quantity $f\left(x^{N} P_{r}(x)\right)$ is proportional to the sum of the weights of all $\mathcal{C}$-partial Motzkin paths of length $N$ which start at height 0 and end at height $r$.

Now let $\mathcal{C}=\xi D+E$ be the matrix from Theorem 6.1, we have that $\mathcal{C}=$ $\frac{1}{1-q} \sqrt{\xi}\left(\sqrt{\xi} \mathrm{d}+\frac{1}{\sqrt{\xi}} \mathrm{e}+\left(\sqrt{\xi}+\frac{1}{\sqrt{\xi}}\right) \mathbb{1}\right)$. Using the arguments of CSSW12, Proposition 2.7] together with [Vie85, Proposition 17, Chapter 1] and Theorem 6.1 yields the result.

From Corollary 6.2, one can compute asymptotics, including the particle current and the particle densities. These quantities were originally computed by Uchiyama [Uch08, and were later recomputed by Cantini [Can17] when $r=N / k$ and $N \rightarrow \infty$. As expected, the phase diagram has three phases: low density, high density and maximal current.

From Corollary 6.2, it is also possible to generalize [CSSW12, Theorem 1.13] and get a closed formula for $Z_{N, r}(\xi)$. This was communicated to us by Dennis Stanton [Sta]. To state this formula, let $(a ; q)_{n}=\prod_{i=0}^{n-1}\left(1-a q^{i}\right),\left(a_{1}, \ldots, a_{k} ; q\right)_{n}=$ $\prod_{i=1}^{k}\left(a_{i} ; q\right)_{n}$ and

Then let

$$
\left[\begin{array}{c}
k \\
r
\end{array}\right]=\frac{(q ; q)_{n}}{(q ; q)_{k}(q ; q)_{n-k}}
$$

$F_{k, r}=(-a / \sqrt{\xi})^{r} q^{\left(\begin{array}{c}r \\ 2\end{array}\right)}\left[\begin{array}{l}k \\ r\end{array}\right]\left(a b, a c / \xi, a d, b c, b d \xi, c d, q, a b c d q^{r-1} ; q\right)_{r} \frac{\left(a b q^{r}, a c q^{r} / \xi, a d q^{r} ; q\right)_{k-r}}{(a b c d q ; q)_{k+r}}$.

and

$$
G_{N, r}(\xi)=\sum_{k=r}^{N} \sum_{j=0}^{k} \frac{F_{k, r} q^{k-j^{2}} a^{-2 j}\left(\xi+1+a q^{j}+\frac{\xi}{a q^{j}}\right)^{N}}{\left(q, \xi q^{1-2 j} / a^{2} ; q\right)_{j}\left(q ; q^{2 j+1} a^{2} / \xi, q ; q\right)_{k-j}}
$$


Theorem 6.3. [Sta] The fugacity partition function is equal to

$$
Z_{N, r}(\xi)=\frac{\prod_{i=2 r}^{N+r-1}\left(\alpha \beta-\gamma \delta q^{i}\right)}{(1-q)^{N-r}} \frac{G_{N, r}(\xi)}{G_{r, r}(\xi)}
$$

using the change of variables from (41), (5), (6) (7).

Proof. The case $r=0$ is [CSSW12, Theorem 1.13], and the proof of this theorem is analogous to the proof there.

Now we return to the proof of Theorem 6.1. We will actually prove a refinement of this theorem; to state it, we need to introduce some notation. Given a word $X$ in $\{D, E\}^{N}$, let $S_{r}(X)$ be the set of words which can be obtained from $X$ by replacing precisely $r$ letters in $X$ by an $A$. Given a word $Z \in S_{r}(X)$, let $D(Z)$ (respectively, $E(Z)$ ) be the set of positions of letters that were $D$ (respectively, $E$ ) in $X$ and became $A$ in $Z$. Let

$$
\operatorname{inv}_{E}(Z)=\sum_{j \in E(Z)}|\{i \in D(Z) \cup E(Z) \mid i<j\}| .
$$

Theorem 6.4. Let $\tilde{\rho}_{r}=\alpha^{r}(1-q)^{r}$. For any word $X$ in $\{D, E\}^{N}$, we have that

$$
\left\langle W|X| V^{r}\right\rangle \cdot \tilde{\rho}_{r}=\sum_{Z \in S_{r}(X)} q^{\operatorname{inv}_{E}(Z)} \alpha^{|D(Z)|} \gamma^{|E(Z)|} \frac{\langle W|Z| V\rangle}{\left\langle W\left|A^{r}\right| V\right\rangle} .
$$

We next explain how Theorem 6.4 refines Theorem 6.1.

Lemma 6.5. Theorem 6.4 implies Theorem 6.1.

Proof. Define $|X|_{D}$ to be the number of $D$ 's that occur in a word $X$. We take (23) and sum it over all words $X$ in $\{D, E\}^{N}$, keeping track of the number of $D$ 's in $X$, and obtaining

$$
\sum_{X \in\{D, E\}^{N}} \xi^{|X|_{D}}\left\langle W|X| V^{r}\right\rangle \cdot \tilde{\rho}_{r}=\sum_{X \in\{D, E\}^{N}} \sum_{Z \in S_{r}(X)} \xi^{|X|_{D}} q^{\operatorname{inv}_{E}(Z)} \alpha^{|D(Z)|} \gamma^{|E(Z)|} \frac{\langle W|Z| V\rangle}{\left\langle W\left|A^{r}\right| V\right\rangle} .
$$

On the left-hand side of (24), we obtain $\left\langle W\left|(\xi D+E)^{N}\right| V^{r}\right\rangle \cdot \tilde{\rho}_{r}=\langle W|(\xi D+$ $E)^{N}\left|V^{r}\right\rangle \cdot \rho_{r} \cdot \prod_{i=0}^{r-1}\left(\alpha \xi+q^{i} \gamma\right)$.

Now we will analyze the right-hand side of (24). Let us fix a word $Z \in\{D, E, A\}^{N}$ which contains precisely $r A^{\prime}$ 's, and determine the coefficient of $\frac{\langle W|Z| V\rangle}{\left\langle W\left|A^{r}\right| V\right\rangle}$. Note that $|X|_{D}=|Z|_{D}+D(Z)$. Also, $\frac{\langle W|Z| V\rangle}{\left\langle W\left|A^{r}\right| V\right\rangle}$ will appear $2^{r}$ times on the right-hand side this is the number of ways that each of those $A$ 's in $Z$ could have come from a $D$ or an $E$ in a word $X \in\{D, E\}^{N}$. Now note that the statistics $E(Z),|D(Z)|,|E(Z)|$ only depend on the substring of $r A$ 's in $Z$, and which letter each of those $A$ 's originally corresponded to in $X$. Let us consider each of the $A$ 's in $Z$ from left to right. If the first $A$ came from a $D$ (respectively, an $E$ ), then we pick up a factor of $\alpha \xi$ (respectively, $\gamma$ ) in $\xi^{|X|_{D}} q^{\operatorname{inv}_{E}(Z)} \alpha^{|D(Z)|} \gamma^{|E(Z)|}$. If the second $A$ came from a $D$ (respectively, an $E$ ), then we pick up a factor of $\alpha \xi$ (respectively, $q \gamma$ ) 
in $\xi^{|X|_{D}} q^{\operatorname{inv}_{E}(Z)} \alpha^{|D(Z)|} \gamma^{|E(Z)|}$ - the $q$ comes from the inversion between the first and second $A$. And in general, if the $i$ th $A$ came from a $D$ (respectively, an $E$ ), then we pick up a factor of $\alpha \xi$ (respectively, $q^{i-1} \gamma$ ). Therefore the coefficient of $\frac{\langle W|Z| V\rangle}{\left\langle W\left|A^{r}\right| V\right\rangle}$ on the right-hand side of (24) is $(\alpha \xi+\gamma)(\alpha \xi+q \gamma) \ldots\left(\alpha \xi+q^{r-1} \gamma\right)$. It follows that the right-hand side equals $\left[y^{r}\right] \frac{\left\langle W\left|(D \xi+E+y A)^{N}\right| V\right\rangle}{\left\langle W\left|A^{r}\right| V\right\rangle} \cdot \prod_{i=0}^{r-1}\left(\alpha \xi+q^{i} \gamma\right)$.

Comparing our expressions for the left-hand side and right-hand side yields (21).

Theorem 6.6. To prove Theorem 6.4, it suffices to prove Theorem 6.4 in the case that $X=D^{N}$. In other words, it suffices to prove that

$$
\left\langle W\left|D^{N}\right| V^{r}\right\rangle \cdot \tilde{\rho}_{r}=\alpha^{r} \sum_{Z \in S_{r}\left(D^{N}\right)} \frac{\langle W|Z| V\rangle}{\left\langle W\left|A^{r}\right| V\right\rangle}=\left[y^{r}\right] \frac{\left\langle W\left|(D+y \alpha A)^{N}\right| V\right\rangle}{\left\langle W\left|A^{r}\right| V\right\rangle} .
$$

Note that the second equality of (25) is obvious. Theorem 6.6 is a consequence of the following two lemmas.

Lemma 6.7. If Theorem 6.4 is true for all words $X$ of the form $E^{\ell} D^{m}$, then it is true for all words $X$ in the letters $D$ and $E$.

Proof. Let $U$ be an arbitrary word in $D$ and $E$. By Corollary 3.7, the matrices $D$, $E$, and $A$ satisfy the relations of Theorem 3.4. In particular we have that $D E=$ $q E D+D+E$. Note that by repeatedly applying the relation $D E=q E D+D+E$ (to replace instances of $D E$ by $q E D+D+E$ ), we can put $U$ into "normal form" that is, we can write $U$ as a linear combination of words of the form $E^{\ell} D^{m}$. We will prove Lemma 6.7 by induction on the number of times one must apply the relation $D E=q E D+D+E$ to put $U$ into normal form. The base case is the words which already have the form $E^{\ell} D^{m}$.

For the inductive step, let us write $U=X D E Y$, where $X$ and $Y$ are words in $D$ and $E$. We have that $X D E Y=q X E D Y+X D Y+X E Y$. By the induction hypothesis, Theorem 6.4 is true for each of the words $X E D Y, X D Y$, and $X E Y$. We want to show that (23) also holds for $X D E Y$.

Let us first analyze the right-hand side of (23) when applied to the word $U=$ $X D E Y$. We will write each word $Z \in S_{r}(X D E Y)$ as $Z_{X} D E Z_{Y}$ or $Z_{X} A E Z_{Y}$ or $Z_{X} D A Z_{Y}$ or $Z_{X} A A Z_{Y}$, where $Z_{X}$ and $Z_{Y}$ have been obtained from $X$ and $Y$, respectively, by replacing some of the letters by $A$ 's. We will also write $Z_{X} Z_{Y}$ to denote the word obtained from one of the four words above by deleting the two letters between $Z_{X}$ and $Z_{Y}$. 
We have that $\sum_{Z \in S_{r}(U)} q^{\operatorname{inv}_{E}(Z)} \alpha^{|D(Z)|} \gamma^{|E(Z)|} \frac{\langle W|Z| V\rangle}{\left\langle W\left|A^{r}\right| V\right\rangle}$ is equal to

$$
\begin{aligned}
& \sum_{Z=Z_{X} D E Z_{Y} \in S_{r}(U)} q^{\operatorname{inv}_{E}\left(Z_{X} Z_{Y}\right)} \alpha^{\left|D\left(Z_{X} Z_{Y}\right)\right|} \gamma^{\left|E\left(Z_{X} Z_{Y}\right)\right|} \frac{\langle W|Z| V\rangle}{\left\langle W\left|A^{r}\right| V\right\rangle} \\
+ & \sum_{Z=Z_{X} A E Z_{Y} \in S_{r}(U)} q^{\left|E\left(Z_{Y}\right)\right|+\operatorname{inv}_{E}\left(Z_{X} Z_{Y}\right)} \alpha^{\left|D\left(Z_{X} Z_{Y}\right)\right|+1} \gamma^{\left|E\left(Z_{X} Z_{Y}\right)\right|} \frac{\langle W|Z| V\rangle}{\left\langle W\left|A^{r}\right| V\right\rangle} \\
+ & \sum_{Z=Z_{X} D A Z_{Y} \in S_{r}(U)} q^{\left|E\left(Z_{Y}\right)\right|+\left|D\left(Z_{X}\right)\right|+\left|E\left(Z_{X}\right)\right|+\operatorname{inv}_{E}\left(Z_{X} Z_{Y}\right)} \alpha^{\left|D\left(Z_{X} Z_{Y}\right)\right|} \gamma^{\left|E\left(Z_{X} Z_{Y}\right)\right|+1} \frac{\langle W|Z| V\rangle}{\left\langle W\left|A^{r}\right| V\right\rangle} \\
+ & \sum_{Z=Z_{X} A A Z_{Y} \in S_{r}(U)} q^{2\left|E\left(Z_{Y}\right)\right|+\left|D\left(Z_{X}\right)\right|+\left|E\left(Z_{X}\right)\right|+1+\operatorname{inv}_{E}\left(Z_{X} Z_{Y}\right)} \alpha^{\left|D\left(Z_{X} Z_{Y}\right)\right|+1} \gamma^{\left|E\left(Z_{X} Z_{Y}\right)\right|+1} \frac{\langle W|Z| V\rangle}{\left\langle W\left|A^{r}\right| V\right\rangle} .
\end{aligned}
$$

Note that in the first three terms above, where $Z$ has the form $Z_{X} D E Z_{Y}, Z_{X} A E Z_{Y}$, and $Z_{X} D A Z_{Y}$, we can apply the relations $D E=q E D+D+E, A E=q E A+A$, and $D A=q A D+A$, to rewrite $\langle W|Z| V\rangle$ as $\left\langle W\left|Z_{X}(q E D+D+E) Z_{Y}\right| V\right\rangle,\langle W| Z_{X}(q E A+$ A) $Z_{Y}|V\rangle$, and $\left\langle W\left|Z_{X}(q A D+A) Z_{Y}\right| V\right\rangle$, respectively.

Now let us analyze the left-hand side of (23) when applied to the word $U=$ $X D E Y$. Applying the re-writing rule $D E=q E D+D+E$ and using the induction hypothesis, we have that $\left\langle W|X(D E) Y| V^{r}\right\rangle \cdot \tilde{\rho}_{r}$ is equal to

$$
\begin{aligned}
& \left(q\left\langle W|X(E D) Y| V^{r}\right\rangle+\left\langle W|X D Y| V^{r}\right\rangle+\left\langle W|X E Y| V^{r}\right\rangle\right) \cdot \tilde{\rho}_{r} \\
= & \sum_{Z \in S_{r}(X E D Y)} q^{\operatorname{inv}_{E}(Z)+1} \alpha^{|D(Z)|} \gamma^{|E(Z)|} \frac{\langle W|Z| V\rangle}{\left\langle W\left|A^{r}\right| V\right\rangle}+\sum_{Z \in S_{r}(X D Y)} q^{\operatorname{inv}_{E}(Z)} \alpha^{|D(Z)|} \gamma^{|E(Z)|} \frac{\langle W|Z| V\rangle}{\left\langle W\left|A^{r}\right| V\right\rangle} \\
& +\sum_{Z \in S_{r}(X E Y)} q^{\operatorname{inv}_{E}(Z)} \alpha^{|D(Z)|} \gamma^{|E(Z)|} \frac{\langle W|Z| V\rangle}{\left\langle W\left|A^{r}\right| V\right\rangle}
\end{aligned}
$$




$$
\begin{aligned}
& =\sum_{Z=Z_{X} E D Z_{Y} \in S_{r}(X E D Y)} q^{\operatorname{inv}_{E}\left(Z_{X} Z_{Y}\right)+1} \alpha^{\left|D\left(Z_{X} Z_{Y}\right)\right|} \gamma^{\left|E\left(Z_{X} Z_{Y}\right)\right|} \frac{\langle W|Z| V\rangle}{\left\langle W\left|A^{r}\right| V\right\rangle} \\
& +\sum_{Z=Z_{X} E A Z_{Y} \in S_{r}(X E D Y)} q^{\left|E\left(Z_{Y}\right)\right|+1+\operatorname{inv}_{E}\left(Z_{X} Z_{Y}\right)} \alpha^{\left|D\left(Z_{X} Z_{Y}\right)\right|+1} \gamma^{\left|E\left(Z_{X} Z_{Y}\right)\right|} \frac{\langle W|Z| V\rangle}{\left\langle W\left|A^{r}\right| V\right\rangle} \\
& +\sum_{Z=Z_{X} A D Z_{Y} \in S_{r}(X E D Y)} q^{\left|D\left(Z_{X}\right)\right|+\left|E\left(Z_{X}\right)\right|+\left|E\left(Z_{Y}\right)\right|+1+\operatorname{inv}_{E}\left(Z_{X} Z_{Y}\right)} \alpha^{\left|D\left(Z_{X} Z_{Y}\right)\right|} \gamma^{\left|E\left(Z_{X} Z_{Y}\right)\right|+1} \frac{\langle W|Z| V\rangle}{\left\langle W\left|A^{r}\right| V\right\rangle} \\
& +\sum_{Z=Z_{X}} \sum_{A A Z_{Y} \in S_{r}(X E D Y)} q^{\left|D\left(Z_{X}\right)\right|+\left|E\left(Z_{X}\right)\right|+2\left|E\left(Z_{Y}\right)\right|+1+\operatorname{inv}_{E}\left(Z_{X} Z_{Y}\right)} \alpha^{\left|D\left(Z_{X} Z_{Y}\right)\right|+1} \gamma^{\left|E\left(Z_{X} Z_{Y}\right)\right|+1} \frac{\langle W|Z| V\rangle}{\left\langle W\left|A^{r}\right| V\right\rangle} \\
& +\sum_{Z=Z_{X} D Z_{Y} \in S_{r}(X D Y)} q^{\operatorname{inv}_{E}\left(Z_{X} Z_{Y}\right)} \alpha^{\left|D\left(Z_{X} Z_{Y}\right)\right|} \gamma^{\left|E\left(Z_{X} Z_{Y}\right)\right|} \frac{\langle W|Z| V\rangle}{\left\langle W\left|A^{r}\right| V\right\rangle}
\end{aligned}
$$

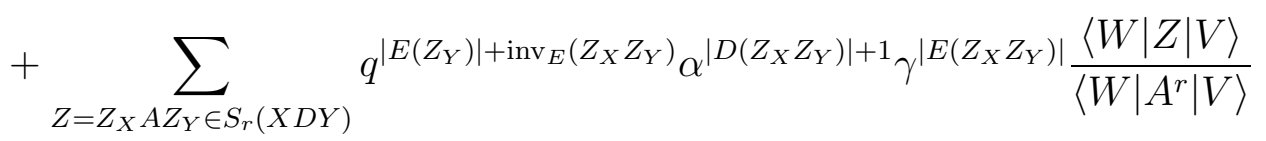

$$
\begin{aligned}
& +\sum_{Z=Z_{X} E Z_{Y} \in S_{r}(X E Y)} q^{\operatorname{inv}_{E}\left(Z_{X} Z_{Y}\right)} \alpha^{\left|D\left(Z_{X} Z_{Y}\right)\right|} \gamma^{\left|E\left(Z_{X} Z_{Y}\right)\right|} \frac{\langle W|Z| V\rangle}{\left\langle W\left|A^{r}\right| V\right\rangle} \\
& +\sum_{Z=Z_{X} A Z_{Y} \in S_{r}(X E Y)} q^{\left|D\left(Z_{X}\right)\right|+\left|E\left(Z_{X}\right)\right|+\left|E\left(Z_{Y}\right)\right|+\operatorname{inv}_{E}\left(Z_{X} Z_{Y}\right)} \alpha^{\left|D\left(Z_{X} Z_{Y}\right)\right|} \gamma^{\left|E\left(Z_{X} Z_{Y}\right)\right|+1} \frac{\langle W|Z| V\rangle}{\left\langle W\left|A^{r}\right| V\right\rangle} .
\end{aligned}
$$

Now if we compare our expressions for the left-hand side and right-hand side of (23), we see that they are equal. This completes the proof.

Lemma 6.8. If Theorem 6.4 is true for all words $X$ of the form $D^{N}$, then it is true for all words $X$ of the form $E^{\ell} D^{m}$.

Proof. We will prove that Theorem 6.4 is true for any word $U$ containing exactly $\ell$ $E$ 's by induction on $\ell$ and on $n$, the number of applications of $D E=q E D+D+E$ which are necessary to put the word in normal form.

If $U$ has the form $X(D E) Y$, then the proof of Lemma 6.7 shows that the theorem holds for $U$, by rewriting $U=X(D E) Y=q X(E D) Y+X D Y+X E Y$. (Note that the process of rewriting does not increase the number of $E$ 's in the word.)

Otherwise we can assume that $U$ has the form $U=E^{\ell} D^{m}$. Let us first analyze the left-hand side of (23) for the word $U$. By Corollary 3.7 and Theorem 3.4, we have the relation $\langle W| E=\frac{1}{\alpha}\langle W|+\frac{\gamma}{\alpha}\langle W| D$. 
Using the induction hypothesis, we have that $\left\langle W\left|E^{\ell} D^{m}\right| V^{r}\right\rangle \cdot \tilde{\rho}_{r}$ is equal to

$$
\begin{aligned}
& \left(\frac{1}{\alpha}\left\langle W\left|E^{\ell-1} D^{m}\right| V^{r}\right\rangle+\frac{\gamma}{\alpha}\left\langle W\left|D E^{\ell-1} D^{m}\right| V^{r}\right\rangle\right) \cdot \tilde{\rho}_{r} \\
& =\sum_{Z \in S_{r}\left(E^{\ell-1} D^{m}\right)} q^{\operatorname{inv}_{E}(Z)} \alpha^{|D(Z)|-1} \gamma^{|E(Z)|} \frac{\langle W|Z| V\rangle}{\left\langle W\left|A^{r}\right| V\right\rangle} \\
& \quad+\sum_{Z=D Z_{X} \in S_{r}\left(D E^{\ell-1} D^{m}\right)} q^{\operatorname{inv}_{E}\left(Z_{X}\right)} \alpha^{\left|D\left(Z_{X}\right)\right|-1} \gamma^{\left|E\left(Z_{X}\right)\right|+1} \frac{\langle W|Z| V\rangle}{\left\langle W\left|A^{r}\right| V\right\rangle} \\
& \quad+\sum_{Z=A Z_{X} \in S_{r}\left(D E^{\ell-1} D^{m}\right)} q^{\left|E\left(Z_{X}\right)\right|+\operatorname{inv}_{E}(Z)} \alpha^{\left|D\left(Z_{X}\right)\right|} \gamma^{\left|E\left(Z_{X}\right)\right|+1} \frac{\langle W|Z| V\rangle}{\left\langle W\left|A^{r}\right| V\right\rangle} .
\end{aligned}
$$

Analyzing the right-hand side of (23) for the word $U$, and again using the relation $\langle W| E=\frac{1}{\alpha}\langle W|+\frac{\gamma}{\alpha}\langle W| D$, we have that

$$
\begin{gathered}
\sum_{Z=E Z_{X} \in S_{r}\left(E^{\ell} D^{m}\right)} q^{\operatorname{inv}_{E}\left(Z_{X}\right)} \alpha^{\left|D\left(Z_{X}\right)\right|} \gamma^{\left|E\left(Z_{X}\right)\right|} \frac{\langle W|Z| V\rangle}{\left\langle W\left|A^{r}\right| V\right\rangle} \\
+\sum_{Z=A Z_{X} \in S_{r}\left(E^{\ell} D^{m}\right)} q^{\left|E\left(Z_{X}\right)\right|+\operatorname{inv}_{E}\left(Z_{X}\right)} \alpha^{\left|D\left(Z_{X}\right)\right|} \gamma^{\left|E\left(Z_{X}\right)\right|+1} \frac{\langle W|Z| V\rangle}{\left\langle W\left|A^{r}\right| V\right\rangle} \\
=\sum_{Z=E Z_{X} \in S_{r}\left(E^{\ell} D^{m}\right)} q^{\operatorname{inv}_{E}\left(Z_{X}\right)} \alpha^{\left|D\left(Z_{X}\right)\right|} \gamma^{\left|E\left(Z_{X}\right)\right|} \frac{\frac{1}{\alpha}\left\langle W\left|Z_{X}\right| V\right\rangle+\frac{\gamma}{\alpha}\left\langle W\left|D Z_{X}\right| V\right\rangle}{\left\langle W\left|A^{r}\right| V\right\rangle} \\
+\sum_{Z=A Z_{X} \in S_{r}\left(E^{\ell} D^{m}\right)} q^{\left|E\left(Z_{X}\right)\right|+\operatorname{inv}_{E}\left(Z_{X}\right)} \alpha^{\left|D\left(Z_{X}\right)\right|} \gamma^{\left|E\left(Z_{X}\right)\right|+1} \frac{\langle W|Z| V\rangle}{\left\langle W\left|A^{r}\right| V\right\rangle} .
\end{gathered}
$$

Comparing our expressions for the left-hand side and right-hand side of (23), we see that they are equal. This completes the proof.

Theorem 6.9. The equation (25) holds. More specifically,

$$
\left\langle W\left|D^{N}\right| V^{r}\right\rangle \cdot \tilde{\rho}_{r}=\left[y^{r}\right] \frac{\left\langle W\left|(D+y \alpha A)^{N}\right| V\right\rangle}{\left\langle W\left|A^{r}\right| V\right\rangle} .
$$

Section 7 will be devoted to the proof of Theorem 6.9. Note that once we have proved Theorem 6.9, this theorem together with Theorem 6.6 will imply Theorem 6.4. And then Theorem 6.4 and Lemma 6.5 will imply that Theorem 6.1 holds.

\section{The proof of Theorem 6.9.}

In this section we will prove Theorem 6.9. We will primarily work with the matrices $\mathrm{d}$ and e defined in Section 3.4, so we begin by writing down the relations satisfied by these matrices.

Lemma 7.1. Let $\mathrm{d}$ and e be defined as in Section 3.4; let $D, E,\langle W|$, and $|V\rangle$ be defined as in Lemma 3.6; let $A=D E-E D$; and let $\left|V^{r}\right\rangle=(0, \ldots, 0,1,0,0, \ldots)^{T}$, 
where the 1 is in the rth position, so that $\left|V^{0}\right\rangle=|V\rangle$. Then we have the following relations.

$$
\begin{aligned}
\mathrm{d} A & =q A \mathrm{~d} \\
A \mathrm{e} & =q \mathrm{e} A \\
\mathrm{de} & =q \mathrm{ed}+(1-q) 1 \\
\mathrm{~d}^{k} \mathrm{e} & =q^{k} \mathrm{ed}^{k}+\left(1-q^{k}\right) \mathrm{d}^{k-1} \\
\mathrm{~d}|V\rangle & =(b+d)|V\rangle-b d \mathrm{e}|V\rangle \\
\langle W| \mathrm{e} & =(a+c)\langle W|-a c\langle W| \mathrm{d} \\
\mathrm{d}\left|V^{k}\right\rangle & =d_{k-1}^{\sharp}\left|V^{k-1}\right\rangle+d_{k}^{\natural}\left|V^{k}\right\rangle+d_{k}^{b}\left|V^{k+1}\right\rangle .
\end{aligned}
$$

Proof. This is a straightforward exercise; most relations follow immediately from the corresponding relations that $D$ and $E$ satisfy.

Let $[k]:=1+q+\cdots+q^{k-1}$ be the $q$-analogue of the number $k$, and let $\left[\begin{array}{c}N \\ r\end{array}\right]_{q}=$ $\frac{[N]_{q} !}{[r]_{q} ![N-r]_{q} !}$.

Theorem 7.2. We have that

$$
\left\langle W\left|\mathrm{~d}^{N}\right| V^{r}\right\rangle=\left[y^{r}\right] \frac{\left\langle W\left|(\mathrm{~d}+y A)^{N}\right| V\right\rangle}{\left\langle W\left|A^{r}\right| V\right\rangle}=\left[\begin{array}{c}
N \\
r
\end{array}\right]_{q} \frac{\left\langle W\left|A^{r} \mathrm{~d}^{N-r}\right| V\right\rangle}{\left\langle W\left|A^{r}\right| V\right\rangle} .
$$

Lemma 7.3. Theorem 7.2 implies Theorem 6.9. 
Proof. Suppose that Theorem 7.2 is true. Then

$$
\begin{aligned}
\left\langle W\left|D^{N}\right| V^{r}\right\rangle \cdot \tilde{\rho}_{r} & =\left\langle W\left|\frac{(1+\mathrm{d})^{N}}{(1-q)^{N}}\right| V^{r}\right\rangle \cdot \alpha^{r}(1-q)^{r} \\
& =\alpha^{r}(1-q)^{r-N} \sum_{i=0}^{N}\left(\begin{array}{c}
N \\
i
\end{array}\right)\left\langle W\left|\mathrm{~d}^{i}\right| V^{r}\right\rangle \\
& =\alpha^{r}(1-q)^{r-N} \sum_{i=0}^{N}\left(\begin{array}{c}
N \\
i
\end{array}\right)\left[y^{r}\right] \frac{\left\langle W\left|(\mathrm{~d}+y A)^{i}\right| V\right\rangle}{\left\langle W\left|A^{r}\right| V\right\rangle} \\
& =\frac{\alpha^{r}(1-q)^{r-N}}{\left\langle W\left|A^{r}\right| V\right\rangle} \sum_{i=0}^{N}\left(\begin{array}{c}
N \\
i
\end{array}\right)\left[y^{r}\right]\left\langle W\left|[(1-q) D-1+y A]^{i}\right| V\right\rangle \\
& =\frac{\alpha^{r}(1-q)^{r-N}}{\left\langle W\left|A^{r}\right| V\right\rangle}\left[y^{r}\right]\left\langle W\left|\sum_{i=0}^{N}\left(\begin{array}{c}
N \\
i
\end{array}\right)[(1-q) D-1+y A]^{i}\right| V\right\rangle \\
& =\frac{\alpha^{r}(1-q)^{r-N}}{\left\langle W\left|A^{r}\right| V\right\rangle}\left[y^{r}\right]\left\langle W\left|[(1-q) D+y A]^{N}\right| V\right\rangle \\
& =\frac{\alpha^{r}}{\left\langle W\left|A^{r}\right| V\right\rangle}\left[y^{r}\right]\left\langle W\left|(D+y A)^{N}\right| V\right\rangle \\
& =\left[y^{r}\right] \frac{(D+y \alpha A)^{N}|V\rangle}{\left\langle W\left|A^{r}\right| V\right\rangle} .
\end{aligned}
$$

To prove Theorem [7.2, we will find an explicit formula for the quantity in (26) (see Corollary (7.10), and use recurrences to show that both $\left\langle W\left|\mathrm{~d}^{N}\right| V^{r}\right\rangle$ and $\left[\begin{array}{c}N \\ r\end{array}\right]_{q} \frac{\left\langle W\left|A^{r} \mathrm{~d}^{N-r}\right| V\right\rangle}{\left\langle W\left|A^{r}\right| V\right\rangle}$ are equal to this explicit formula. Note that the second equality in Theorem 7.2 follows by repeated application of the relation $\mathrm{d} A=q A \mathrm{~d}$.

7.1. A formula for $\left\langle W\left|A^{r} \mathrm{~d}^{N-r}\right| V\right\rangle$. First we will prove a recurrence for $\left\langle W\left|A^{r} \mathrm{~d}^{m}\right| V\right\rangle$ (where we are thinking of $m=N-r$ ).

Proposition 7.4. We have that

$$
\left\langle W\left|A^{r} \mathrm{~d}^{m}\right| V\right\rangle=\frac{b+d-b d(a+c) q^{m+r-1}}{1-a b c d q^{m+2 r-1}}\left\langle W\left|A^{r} \mathrm{~d}^{m-1}\right| V\right\rangle+\frac{b d\left(q^{m-1}-1\right)}{1-a b c d q^{m+2 r-1}}\left\langle W\left|A^{r} \mathrm{~d}^{m-2}\right| V\right\rangle .
$$


Proof. In what follows, we will use the relations from Lemma 7.1. Note that

$$
\begin{aligned}
\left\langle W\left|A^{r} \mathrm{~d}^{m}\right| V\right\rangle= & \left\langleW \left| A^{r} \mathrm{~d}^{m-1}((b+d)|V\rangle-b d \mathrm{e}|V\rangle)\right.\right. \\
= & (b+d)\left\langle W\left|A^{r} \mathrm{~d}^{m-1}\right| V\right\rangle-b d\left\langle W\left|A^{r} \mathrm{~d}^{m-1} \mathrm{e}\right| V\right\rangle \\
= & (b+d)\left\langle W\left|A^{r} \mathrm{~d}^{m-1}\right| V\right\rangle-b d\left(q^{m-1}\left\langle W\left|A^{r} \mathrm{ed}^{m-1}\right| V\right\rangle-\left(q^{m-1}-1\right)\left\langle W\left|A^{r} \mathrm{~d}^{m-2}\right| V\right\rangle\right) \\
= & (b+d)\left\langle W\left|A^{r} \mathrm{~d}^{m-1}\right| V\right\rangle-b d q^{m-1}\left\langle W\left|A^{r} \mathrm{ed}^{m-1}\right| V\right\rangle+b d\left(q^{m-1}-1\right)\left\langle W\left|A^{r} \mathrm{~d}^{m-2}\right| V\right\rangle \\
= & (b+d)\left\langle W\left|A^{r} \mathrm{~d}^{m-1}\right| V\right\rangle-b d q^{m+r-1}\left\langle W\left|\mathrm{e} A^{r} \mathrm{~d}^{m-1}\right| V\right\rangle+b d\left(q^{m-1}-1\right)\left\langle W\left|A^{r} \mathrm{~d}^{m-2}\right| V\right\rangle \\
= & (b+d)\left\langle W\left|A^{r} \mathrm{~d}^{m-1}\right| V\right\rangle-b d q^{m+r-1}\left[(a+c)\left\langle W\left|A^{r} \mathrm{~d}^{m-1}\right| V\right\rangle-a c\left\langle W\left|\mathrm{~d} A^{r} \mathrm{~d}^{m-1}\right| V\right\rangle\right] \\
& \quad+b d\left(q^{m-1}-1\right)\left\langle W\left|A^{r} \mathrm{~d}^{m-2}\right| V\right\rangle \\
= & (b+d)\left\langle W\left|A^{r} \mathrm{~d}^{m-1}\right| V\right\rangle-b d(a+c) q^{m+r-1}\left\langle W\left|A^{r} \mathrm{~d}^{m-1}\right| V\right\rangle+a b c d q^{m+2 r-1}\left\langle W\left|A^{r} \mathrm{~d}^{m}\right| V\right\rangle \\
& \quad+b d\left(q^{m-1}-1\right)\left\langle W\left|A^{r} \mathrm{~d}^{m-2}\right| V\right\rangle .
\end{aligned}
$$

Our next goal is to get an explicit formula for $\frac{\left\langle W\left|A^{r} \mathrm{~d}^{m}\right| V\right\rangle}{\left\langle W\left|A^{r}\right| V\right\rangle}$ (see Corollary 17.10). Towards this end, we make the following definition.

Definition 7.5. Define $F_{m}(y)$ by $F_{0}(y)=1, F_{m}(y)=0$ if $m<0$, and

$$
\left.F_{m}(y)=\left(b+d-y(a+c) q^{m-1}\right) F_{m-1}(y)\right)+\left(q^{m-1}-1\right)\left(b d-a c q^{m-2} y^{2}\right) F_{m-2}(y)
$$

for $m>0$.

Corollary 7.6. We have that

$$
\frac{\left\langle W\left|A^{r} \mathrm{~d}^{m}\right| V\right\rangle}{\left\langle W\left|A^{r}\right| V\right\rangle}=\frac{F_{m}\left(b d q^{r}\right)}{\prod_{i=0}^{m-1}\left(1-a b c d q^{2 r+i}\right)} .
$$

Proof. Corollary 7.6 follows from Proposition 7.4 and Definition 7.5 by comparing the recurrences and base cases.

Theorem 7.7. Set

$$
B_{m}(b, d)=\sum_{i=0}^{m}\left[\begin{array}{c}
m \\
i
\end{array}\right]_{q} b^{i} d^{m-i} \text { and } A_{m}(a, c)=\sum_{i=0}^{m}\left[\begin{array}{c}
m \\
i
\end{array}\right]_{1 / q} a^{i} c^{m-i}
$$

Then for $m \geq 0$, we have

$$
F_{m}(y)=\sum_{i=0}^{m}(-1)^{i}\left[\begin{array}{c}
m \\
i
\end{array}\right]_{q} q^{\left(\begin{array}{l}
i \\
2
\end{array}\right)} y^{i} A_{i}(a, c) B_{m-i}(b, d) .
$$

Before proving Theorem 7.7, we first state a few useful lemmas. Lemma 7.8 is a simple exercise (and is well-known). 


\section{Lemma 7.8.}

$$
\begin{aligned}
{\left[\begin{array}{c}
m \\
i
\end{array}\right]_{q} } & =\left[\begin{array}{c}
m-1 \\
i
\end{array}\right]_{q}+q^{m-i}\left[\begin{array}{c}
m-1 \\
i-1
\end{array}\right]_{q} \\
{\left[\begin{array}{c}
m \\
i
\end{array}\right]_{q} } & =q^{i}\left[\begin{array}{c}
m-1 \\
i
\end{array}\right]_{q}+\left[\begin{array}{c}
m-1 \\
i-1
\end{array}\right]_{q} \\
\left(1-q^{m}\right)\left[\begin{array}{c}
m-1 \\
i
\end{array}\right]_{q} & =\left(1-q^{m-i}\right)\left[\begin{array}{c}
m \\
i
\end{array}\right]_{q}
\end{aligned}
$$

Lemma 7.9. We have that

$$
\begin{aligned}
& (b+d) B_{m}(b, d)=B_{m+1}(b, d)+\left(1-q^{m}\right) b d B_{m-1}(b, d), \text { and } \\
& (a+c) A_{m}(a, c)=A_{m+1}(a, c)+\left(1-q^{-m}\right) a c A_{m-1}(a, c) .
\end{aligned}
$$

Proof. It suffices to prove the first equation. We will use Lemma 7.8, Note that

$$
\begin{aligned}
(b+d) B_{m}(b, d) & =\sum_{i=0}^{m}\left[\begin{array}{c}
m \\
i
\end{array}\right]_{q} b^{i} d^{m+1-i}+\sum_{i=1}^{m+1}\left[\begin{array}{c}
m \\
i-1
\end{array}\right]_{q} b^{i} d^{m+1-i} \\
& =d^{m+1}+b^{m+1}+\sum_{i=1}^{m}\left(\left[\begin{array}{c}
m \\
i
\end{array}\right]_{q}+\left[\begin{array}{c}
m \\
i-1
\end{array}\right]_{q}\right) b^{i} d^{m+1-i} \\
& =d^{m+1}+b^{m+1}+\sum_{i=1}^{m}\left(\left[\begin{array}{c}
m+1 \\
i
\end{array}\right]_{q}+\left(1-q^{m-i+1}\right)\left[\begin{array}{c}
m \\
i-1
\end{array}\right]_{q} b^{i} d^{m+1-i}\right. \\
& =\sum_{i=0}^{m+1}\left[\begin{array}{c}
m+1 \\
i
\end{array}\right]_{q} b^{i} d^{m+1-i}+\sum_{j=1}^{m}\left(1-q^{m-j+1}\right)\left[\begin{array}{c}
m \\
j-1
\end{array}\right]_{q} b^{j} d^{m+1-j} \\
& =\sum_{i=0}^{m+1}\left[\begin{array}{c}
m+1 \\
i
\end{array}\right]_{q} b^{i} d^{m+1-i}+\sum_{i=0}^{m-1}\left(1-q^{m-i}\right)\left[\begin{array}{c}
m \\
i
\end{array}\right]_{q} b^{i+1} d^{m-i} \\
& =\sum_{i=0}^{m+1}\left[\begin{array}{c}
m+1 \\
i
\end{array}\right]_{q} b^{i} d^{m+1-i}+\left(1-q^{m}\right) \sum_{i=0}^{m-1}\left[\begin{array}{c}
m-1 \\
i
\end{array}\right]_{q} b^{i+1} d^{m-i} \\
& =B_{m+1}(b, d)+\left(1-q^{m}\right) b d B_{m-1}(b, d) .
\end{aligned}
$$

We now prove Theorem 7.7 .

Proof. The case $m=0$ is trivial. We prove the proposition by induction. Suppose that the proposition is true for all $n<m$. Then we have the following (see the end 
of the list of equations for justifications of each line):

$$
\begin{aligned}
& \left(b+d-y(a+c) q^{m-1}\right) F_{m-1}(y) \\
& =\left(b+d-y(a+c) q^{m-1}\right) \sum_{i=0}^{m-1}(-1)^{i}\left[\begin{array}{c}
m-1 \\
i
\end{array}\right]_{q} q^{\left(\begin{array}{c}
i \\
2
\end{array}\right)} y^{i} A_{i}(a, c) B_{m-1-i}(b, d) \\
& =\sum_{i=0}^{m-1}(-1)^{i}\left[\begin{array}{c}
m-1 \\
i
\end{array}\right]_{q} q^{\left(\begin{array}{c}
i \\
2
\end{array}\right)} y^{i} A_{i}(a, c)(b+d) B_{m-1-i}(b, d) \\
& -\sum_{i=0}^{m-1}(-1)^{i}\left[\begin{array}{c}
m-1 \\
i
\end{array}\right]_{q} q^{\left(\begin{array}{c}
i \\
2
\end{array}\right)+m-1} y^{i+1}(a+c) A_{i}(a, c) B_{m-1-i}(b, d) \\
& =\sum_{i=0}^{m}(-1)^{i}\left[\begin{array}{c}
m-1 \\
i
\end{array}\right]_{q} q^{\left(\begin{array}{c}
i \\
2
\end{array}\right)} y^{i} A_{i}(a, c) B_{m-i}(b, d) \\
& +\sum_{i}(-1)^{i}\left[\begin{array}{c}
m-1 \\
i
\end{array}\right]_{q} q^{\left(\begin{array}{c}
i \\
2
\end{array}\right)} y^{i} A_{i}(a, c)\left(1-q^{m-i-1}\right) b d B_{m-i-2}(b, d) \\
& -\sum_{i=0}^{m}(-1)^{i} q^{m-1}\left[\begin{array}{c}
m-1 \\
i
\end{array}\right]_{q} q^{\left(\begin{array}{c}
i \\
2
\end{array}\right)} y^{i+1} A_{i+1}(a, c) B_{m-i-1}(b, d) \\
& -\sum_{i}(-1)^{i} q^{m-1}\left[\begin{array}{c}
m-1 \\
i
\end{array}\right]_{q} q^{\left(\begin{array}{c}
i \\
2
\end{array}\right)} y^{i+1} a c\left(1-q^{-i}\right) A_{i-1}(a, c) B_{m-i-1}(b, d) \\
& =\sum_{i=0}^{m}(-1)^{i}\left[\begin{array}{c}
m-1 \\
i
\end{array}\right]_{q} q^{\left(\begin{array}{c}
i \\
2
\end{array}\right)} y^{i} A_{i}(a, c) B_{m-i}(b, d) \\
& +\sum_{i}(-1)^{i}\left[\begin{array}{c}
m-2 \\
i
\end{array}\right]_{q} q^{\left(\begin{array}{c}
i \\
2
\end{array}\right)} y^{i} A_{i}(a, c)\left(1-q^{m-1}\right) b d B_{m-i-2}(b, d) \\
& -\sum_{j=1}^{m}(-1)^{j-1} q^{m-1}\left[\begin{array}{c}
m-1 \\
j-1
\end{array}\right]_{q} q^{\left(\begin{array}{c}
j-1 \\
2
\end{array}\right)} y^{j} A_{j}(a, c) B_{m-j}(b, d) \\
& +\sum_{i}(-1)^{i} q^{m-1-i}\left[\begin{array}{c}
m-2 \\
i-1
\end{array}\right]_{q} q^{\left(\begin{array}{c}
i \\
2
\end{array}\right)} y^{i+1} a c\left(1-q^{m-1}\right) A_{i-1}(a, c) B_{m-i-1}(b, d) \\
& =\sum_{i=0}^{m}(-1)^{i}\left(\left[\begin{array}{c}
m-1 \\
i
\end{array}\right]_{q}+q^{m-i}\left[\begin{array}{c}
m-1 \\
i-1
\end{array}\right]_{q}\right) q^{\left(\begin{array}{c}
i \\
2
\end{array}\right)} y^{i} A_{i}(a, c) B_{m-i}(b, d) \\
& +\sum_{i}(-1)^{i}\left[\begin{array}{c}
m-2 \\
i
\end{array}\right]_{q} q^{\left(\begin{array}{c}
i \\
2
\end{array}\right)} y^{i} A_{i}(a, c)\left(1-q^{m-1}\right) b d B_{m-i-2}(b, d) \\
& +\sum_{i}(-1)^{i} q^{m-1-i}\left[\begin{array}{c}
m-2 \\
i-1
\end{array}\right]_{q} q^{\left(\begin{array}{c}
i \\
2
\end{array}\right)} y^{i+1} a c\left(1-q^{m-1}\right) A_{i-1}(a, c) B_{m-i-1}(b, d)
\end{aligned}
$$




$$
\begin{aligned}
= & \sum_{i=0}^{m}(-1)^{i}\left[\begin{array}{c}
m \\
i
\end{array}\right]_{q} q^{\left(\begin{array}{c}
i \\
2
\end{array}\right)} y^{i} A_{i}(a, c) B_{m-i}(b, d) \\
& +\left(1-q^{m-1}\right) b d \sum_{i}(-1)^{i}\left[\begin{array}{c}
m-2 \\
i
\end{array}\right]_{q} q^{\left(\begin{array}{c}
i \\
2
\end{array}\right)} y^{i} A_{i}(a, c) B_{m-i-2}(b, d) \\
& -\left(1-q^{m-1}\right) a c y^{2} q^{m-2} \sum_{j}(-1)^{j}\left[\begin{array}{c}
m-2 \\
j
\end{array}\right]_{q} q^{\left(\begin{array}{l}
j \\
2
\end{array}\right)} y^{j} A_{j}(a, c) B_{m-j-2}(b, d) \\
= & \sum_{i=0}^{m}(-1)^{i}\left[\begin{array}{c}
m \\
i
\end{array}\right]_{q} q^{\left(\begin{array}{c}
i \\
2
\end{array}\right)} y^{i} A_{i}(a, c) B_{m-i}(b, d)+\left(1-q^{m-1}\right)\left(b d-a c q^{m-2} y^{2}\right) F_{m-2}(y)
\end{aligned}
$$

For the first equality, we used the induction hypothesis, and for the second equality we simply distributed terms. For the third equality we used Lemma 7.9, For the fourth equality, we changed the index of summation in the third line, and we also used Lemma 7.8:

$$
\left(1-q^{m-i-1}\right)\left[\begin{array}{c}
m-1 \\
i
\end{array}\right]_{q}=\left(1-q^{m-1}\right)\left[\begin{array}{c}
m-2 \\
i
\end{array}\right]_{q}
$$

and

$$
\left(1-q^{i}\right)\left[\begin{array}{c}
m-1 \\
i
\end{array}\right]_{q}=\left(1-q^{m-1}\right)\left[\begin{array}{c}
m-2 \\
i-1
\end{array}\right]_{q}
$$

For the fifth equality, we combined two terms. For the sixth equality, we used Lemma [7.9, and we changed the index of summation in the third line. For the seventh equality, we used the induction hypothesis.

Now we have shown that

$$
\left(b+d-y(a+c) q^{m-1}\right) F_{m-1}(y)
$$

is equal to

$$
\sum_{i=0}^{m}(-1)^{i}\left[\begin{array}{c}
m \\
i
\end{array}\right]_{q} q^{\left(\begin{array}{c}
i \\
2
\end{array}\right)} y^{i} A_{i}(a, c) B_{m-i}(b, d)+\left(1-q^{m-1}\right)\left(b d-a c q^{m-2} y^{2}\right) F_{m-2}(y) .
$$

Comparing this to the defining recurrence for $F_{m}(y)$ (Definition 7.5 ), we have proved the desired explicit formula for $F_{m}(y)$.

Corollary 7.10. We have that

$$
\frac{\left\langle W\left|A^{r} d^{m}\right| V\right\rangle}{\left\langle W\left|A^{r}\right| V\right\rangle}=\frac{\sum_{i=0}^{m}(-1)^{i}\left[\begin{array}{c}
m \\
i
\end{array}\right]_{q} q^{\left(\begin{array}{c}
i \\
2
\end{array}\right)}\left(b d q^{r}\right)^{i} A_{i}(a, c) B_{m-i}(b, d)}{\prod_{i=0}^{m-1}\left(1-a b c d q^{2 r+i}\right)} .
$$

Proof. This follows from Corollary 7.6 and Theorem 7.7. 
7.2. A formula for $\left\langle W\left|\mathrm{~d}^{N}\right| V^{r}\right\rangle$. First we will prove a recurrence for $\left\langle W\left|\mathrm{~d}^{N}\right| V^{r}\right\rangle$. Towards this end, we state a simple lemma, which follows from the definitions of the tridiagonal matrices $\mathrm{d}$ and $\mathrm{e}$.

\section{Lemma 7.11.}

$$
\mathrm{d}\left|V^{r}\right\rangle=\left(1-q^{2 r-1} a b c d\right)\left|V^{r-1}\right\rangle-b d q^{r} \mathrm{e}\left|V^{r}\right\rangle+R_{r}\left|V^{r}\right\rangle
$$

where

$$
R_{r}=\frac{q^{r-1}}{1-a b c d q^{2 r-2}}\left(q(b+d)-q^{r-1}(b+d) a b c d+b d(a+c)\left(1-q^{r}\right)\right) .
$$

Theorem 7.12. The quantity $\left\langle W\left|\mathrm{~d}^{N}\right| V^{r}\right\rangle$ satisfies the following recurrence.

$$
\begin{aligned}
\left(1-a b c d q^{r+N-1}\right)\left\langle W\left|\mathrm{~d}^{N}\right| V^{r}\right\rangle & =\left(1-q^{2 r-1} a b c d\right)\left\langle W\left|\mathrm{~d}^{N-1}\right| V^{r-1}\right\rangle-b d q^{r}\left(1-q^{N-1}\right)\left\langle W\left|\mathrm{~d}^{N-2}\right| V^{r}\right\rangle \\
& +\left(R_{r}-b d q^{r+N-1}(a+c)\right)\left\langle W\left|\mathbf{d}^{N-1}\right| V^{r}\right\rangle .
\end{aligned}
$$

Proof. In what follows, we will use Lemma 7.11 and Lemma 7.1 - in particular the relations $\mathrm{d}^{k} \mathrm{e}=q^{k} \mathrm{ed}^{k}+\left(1-q^{k}\right) \mathrm{d}^{k-1}$ and $\langle W| \mathrm{e}=(a+c)\langle W|-a c\langle W| \mathrm{d}$.

$$
\begin{aligned}
\left\langle W\left|\mathrm{~d}^{N}\right| V^{r}\right\rangle= & \left(1-q^{2 r-1} a b c d\right)\left\langle W\left|\mathrm{~d}^{N-1}\right| V^{r-1}\right\rangle-b d q^{r}\left\langle W\left|\mathrm{~d}^{N-1} \mathrm{e}\right| V^{r}\right\rangle+R_{r}\left\langle W\left|\mathrm{~d}^{N-1}\right| V^{r}\right\rangle \\
= & \left(1-q^{2 r-1} a b c d\right)\left\langle W\left|\mathrm{~d}^{N-1}\right| V^{r-1}\right\rangle+R_{r}\left\langle W\left|\mathrm{~d}^{N-1}\right| V^{r}\right\rangle \\
& -b d q^{r+N-1}\left\langle W\left|\mathrm{ed}^{N-1}\right| V^{r}\right\rangle-b d q^{r}\left(1-q^{N-1}\right)\left\langle W\left|\mathrm{~d}^{N-2}\right| V^{r}\right\rangle \\
= & \left(1-q^{2 r-1} a b c d\right)\left\langle W\left|\mathrm{~d}^{N-1}\right| V^{r-1}\right\rangle+R_{r}\left\langle W\left|\mathrm{~d}^{N-1}\right| V^{r}\right\rangle-b d q^{r+N-1}(a+c)\left\langle W\left|\mathrm{~d}^{N-1}\right| V^{r}\right\rangle \\
& +a b c d q^{r+N-1}\left\langle W\left|\mathrm{~d}^{N}\right| V^{r}\right\rangle-b d q^{r}\left(1-q^{N-1}\right)\left\langle W\left|\mathrm{~d}^{N-2}\right| V^{r}\right\rangle .
\end{aligned}
$$

Collecting like terms now yields the recurrence in Theorem 7.12 .

Theorem 7.13. We have that

$$
\left\langle W\left|\mathrm{~d}^{m+r}\right| V^{r}\right\rangle=\left[\begin{array}{c}
m+r \\
r
\end{array}\right]_{q} \frac{F_{m}\left(b d q^{r}\right)}{\prod_{i=0}^{m-1}\left(1-a b c d q^{2 r+i}\right)} .
$$

Note that once we have proved Theorem 7.13 , combining this theorem with Corollary 7.6 will prove Theorem 7.2 and hence Theorem 6.9.

Proposition 7.14. Define

$$
C(m, r)=\frac{F_{m}\left(b d q^{r}\right)}{\prod_{i=0}^{m-1}\left(1-a b c d q^{2 r+i}\right)} .
$$

Then $C(m, r)$ satisfies the recurrence

$$
\left(1-a b c d q^{2 r+m-1}\right) C(m, r)=A_{1}(m, r) C(m, r-1)-A_{2}(m, r) C(m-2, r)+A_{3}(m, r) C(m-1, r),
$$


where

$$
\begin{aligned}
& A_{1}=\frac{\left(1-q^{2 r-1} a b c d\right)\left(1-q^{r}\right)}{\left(1-q^{m+r}\right)} \\
& A_{2}=\frac{b d q^{r}\left(1-q^{m-1}\right)\left(1-q^{m}\right)}{\left(1-q^{m+r}\right)} \\
& A_{3}=\frac{\left(-b d(a+c) q^{m-1+2 r}+R_{r}\right)\left(1-q^{m}\right)}{\left(1-q^{m+r}\right)} .
\end{aligned}
$$

Equivalently, $F_{m}\left(b d q^{r}\right)$ satisfies the following recurrence.

$$
\begin{aligned}
\left(1-q^{m+r}\right)(1 & \left.-a b c d q^{2 r-2}\right) F_{m}\left(b d q^{r}\right)=\left(1-q^{r}\right)\left(1-a b c d q^{2 r+m-2}\right) F_{m}\left(b d q^{r-1}\right) \\
& -b d q^{r}\left(1-q^{m-1}\right)\left(1-q^{m}\right)\left(1-a b c d q^{2 r-2}\right)\left(1-a b c d q^{2 r+m-2}\right) F_{m-2}\left(b d q^{r}\right) \\
& +\left(1-q^{m}\right)\left(-b d(a+c) q^{2 r+m-1}+R_{r}\right)\left(1-a b c d q^{2 r-2}\right) F_{m-1}\left(b d q^{r}\right) .
\end{aligned}
$$

Lemma 7.15. To prove Theorem 7.13, it suffices to prove Proposition 7.14.

Proof. To prove Theorem 7.13 , we need to show that

$$
\left\langle W\left|\mathrm{~d}^{m+r}\right| V^{r}\right\rangle=\left[\begin{array}{c}
m+r \\
r
\end{array}\right]_{q} C(m, r) .
$$

It is easy to verify this equation for $m+r \leq 1$. Moreover it is straightforward to verify that the recurrences in Proposition 7.14 are equivalent to the recurrence for $\left\langle W\left|\mathrm{~d}^{m+r}\right| V^{r}\right\rangle$ (see Theorem 7.12$)$, provided that we have (32).

Therefore to complete the proof of Theorem 7.13, we need to prove Proposition 7.14. In particular, we will prove the recurrence for $F_{m}\left(b d q^{r}\right)$, which follows from Proposition 7.16 below when $y=q^{r}$.

Proposition 7.16. $F_{m}(b d y)$ satisfies the following recurrence.

$$
\begin{aligned}
\left(1-y q^{m}\right) & \left(1-y^{2} q^{-2} a b c d\right) F_{m}(b d y)=\left(1-y^{2} q^{m-2} a b c d\right)(1-y) F_{m}(b d y / q) \\
& -b d y\left(1-q^{m}\right)\left(1-q^{m-1}\right)\left(1-a b c d y^{2} q^{m-2}\right)\left(1-a b c d y^{2} q^{-2}\right) F_{m-2}(b d y) \\
& +\left(1-q^{m}\right)\left\{y\left[b+d+q^{-1} b d(a+c)\right]-y^{2}\left[q^{-1}\left(1+q^{m}\right) b d(a+c)+q^{-2}(b+d) a b c d\right]\right. \\
& \left.+y^{4}\left[q^{m-3} a b^{2} c d^{2}(a+c)\right]\right\} F_{m-1}(b d y) .
\end{aligned}
$$

We will prove Proposition 7.16 by taking the coefficient of $y^{n}$ in the recurrence and showing that it is an identity. Towards this end, we define

$$
X_{m, n}=(-1)^{n}\left[\begin{array}{c}
m \\
n
\end{array}\right]_{q} q^{\left(\begin{array}{c}
n \\
2
\end{array}\right)} b^{n} d^{n} A_{n}(a, c) B_{m-n}(b, d),
$$

which is the coefficient of $y^{n}$ in $F_{m}(b d y)$. 
Lemma 7.17. Taking the coefficient of $y^{n}$ in the recurrence of Proposition 7.16] yields the following equation.

$$
\begin{aligned}
X_{m, n} & -q^{-2} a b c d X_{m, n-2}-q^{m} X_{m, n-1}+q^{m-2} a b c d X_{m, n-3} \\
& =q^{-n} X_{m, n}-q^{-n+1} X_{m, n-1}-q^{m-n} a b c d X_{m, n-2}+q^{m-n+1} a b c d X_{m, n-3} \\
& -\left(1-q^{m}\right)\left(1-q^{m-1}\right) b d X_{m-2, n-1}+q^{-2}\left(1-q^{m}\right)\left(1-q^{m-1}\right) a b^{2} c d^{2} X_{m-2, n-3} \\
& +\left(1-q^{m}\right)\left(1-q^{m-1}\right)\left[q^{m-2} a b^{2} c d^{2} X_{m-2, n-3}-q^{m-4}(a b c d)^{2} b d X_{m-2, n-5}\right] \\
& +\left(1-q^{m}\right)(b+d) X_{m-1, n-1}+q^{-1}\left(1-q^{m}\right) b d(a+c) X_{m-1, n-1} \\
& -\left(1-q^{m}\right)\left[q^{-1}\left(1+q^{m}\right) b d(a+c)+q^{-2} a b c d(b+d)\right] X_{m-1, n-2} \\
& +\left(1-q^{m}\right) q^{m-3} a c(a+c)(b d)^{2} X_{m-1, n-4} .
\end{aligned}
$$

Proof. The proof is straightforward so we omit it.

Note that the total degree in $a$ and $c$ in $X_{m, n}$ is $n$, while the total degree in $b$ and $d$ in $X_{m, n}$ is $m+n$. Moreover, each term in (34) has one of the following properties:

- the total degree in $a$ and $c$ is $n$, and the total degree in $b$ and $d$ is $m+n$, or

- the total degree in $a$ and $c$ is $n-1$, and the total degree in $b$ and $d$ is $m+n-1$.

So we can split (34) into two equations based on which of these two conditions holds. We get equations (35) and (36), respectively.

$$
\begin{array}{r}
q^{2}\left(1-q^{-n}\right) X_{m, n}-a b c d\left(1-q^{m-n+2}\right) X_{m, n-2}-q\left(1-q^{m}\right) b d(a+c) X_{m-1, n-1} \\
+\left(1-q^{m}\right) a b c d(b+d) X_{m-1, n-2}=0
\end{array}
$$

$$
\begin{gathered}
\left(q^{-n+1}-q^{m}\right) X_{m, n-1}+\left(q^{m-2}-q^{m-n+1}\right) a b c d X_{m, n-3}-\left(1-q^{m}\right)(b+d) X_{m-1, n-1} \\
+q^{-1}\left(1-q^{m}\right)\left(1+q^{m}\right) b d(a+c) X_{m-1, n-2}-q^{m-3}\left(1-q^{m}\right) a c(a+c)(b d)^{2} X_{m-1, n-4} \\
+\left(1-q^{m}\right)\left(1-q^{m-1}\right) b d\left\{X_{m-2, n-1}-\left(q^{-2}+q^{m-2}\right) a b c d X_{m-2, n-3}\right. \\
\left.+q^{m-4}(a b c d)^{2} X_{m-2, n-5}\right\}=0 .
\end{gathered}
$$

Now it is clear that Proposition 7.16 follows from Lemmas 7.18 and 7.19 below. To prove the lemmas, we will take the coefficient of $a^{j} b^{n+i} c^{n-j} d^{m-i}$ in each of (35) and (36), obtaining identities involving $q$-binomial coefficients which can be proved by hand or with any computer algebra system.

Let $x(m, n, i, j)$ denote the coefficient of $a^{j} b^{n+i} c^{n-j} d^{m+n-i}$ in $X_{m, n}(q)$. It is easy to check that

$$
x(m, n, i, j)=(-1)^{n} q^{\left(\begin{array}{c}
n \\
2
\end{array}\right)}\left[\begin{array}{c}
m \\
n
\end{array}\right]_{q}\left[\begin{array}{c}
m-n \\
i
\end{array}\right]_{q}\left[\begin{array}{c}
n \\
j
\end{array}\right]_{1 / q} .
$$

Lemma 7.18. Equation (35) is an identity. 
Proof. It suffices to prove that taking the coefficient of $a^{j} b^{n+i} c^{n-j} d^{m-i}$ in (35) yields an identity. When we take this coefficient we obtain the following equation.

$$
\begin{aligned}
& \left(1-q^{-n}\right) x(m, n, i, j)-\left(q^{-2}-q^{m-n}\right) x(m, n-2, i+1, j-1) \\
& -q^{-1}\left(1-q^{m}\right)[x(m-1, n-1, i, j)+x(m-1, n-1, i, j-1)] \\
& +q^{-2}\left(1-q^{m}\right)[x(m-1, n-2, i+1, j-1)+x(m-1, n-2, i, j-1)]=0 .
\end{aligned}
$$

Using (37) now yields the equation

$$
\begin{aligned}
& q^{\left(\begin{array}{c}
n \\
2
\end{array}\right)\left(1-q^{-n}\right)}\left[\begin{array}{c}
m \\
n
\end{array}\right]_{q}\left[\begin{array}{c}
m-n \\
i
\end{array}\right]_{q}\left[\begin{array}{l}
n \\
j
\end{array}\right]_{1 / q} \\
& -q^{\left(\begin{array}{c}
n-2 \\
2
\end{array}\right)\left(q^{-2}-q^{m-n}\right)}\left[\begin{array}{c}
m \\
n-2
\end{array}\right]_{q}\left[\begin{array}{c}
m-n+2 \\
i+1
\end{array}\right]_{q}\left[\begin{array}{c}
n-2 \\
j-1
\end{array}\right]_{1 / q} \\
& +q^{\left(\begin{array}{c}
n-1 \\
2
\end{array}\right)-1}\left(1-q^{m}\right)\left[\begin{array}{c}
m-1 \\
n-1
\end{array}\right]_{q}\left[\begin{array}{c}
m-n \\
i
\end{array}\right]_{q}\left(\left[\begin{array}{c}
n-1 \\
j-1
\end{array}\right]_{1 / q}+\left[\begin{array}{c}
n-1 \\
j
\end{array}\right]_{1 / q}\right) \\
& +q^{\left(\begin{array}{c}
n-2 \\
2
\end{array}\right)-2}\left(1-q^{m}\right)\left[\begin{array}{c}
m-1 \\
n-2
\end{array}\right]_{q}\left(\left[\begin{array}{c}
m-n+1 \\
i
\end{array}\right]_{q}+\left[\begin{array}{c}
m-n+1 \\
i+1
\end{array}\right]_{q}\right)\left[\begin{array}{c}
n-2 \\
j-1
\end{array}\right]_{1 / q}=0 .
\end{aligned}
$$

Simplifying this, we get the equation

$$
\begin{aligned}
& q^{2 n-1}\left(1-q^{-n}\right)-\frac{q^{n-1}\left(1-q^{m-n+2}\right)\left(1-q^{j}\right)\left(1-q^{n-j}\right)}{\left(1-q^{i+1}\right)\left(1-q^{m-n+1-i}\right)}-q^{2 n-1}\left(2-q^{-j}-q^{-n+j}\right) \\
& +q^{2 n-1}\left(1-q^{-j}\right)\left(1-q^{-n+j}\right)\left(\frac{1}{1-q^{m-n+1-i}}+\frac{1}{1-q^{1+i}}\right)=0
\end{aligned}
$$

which is trivially true.

Lemma 7.19. Equation (36) is an identity.

Proof. We use the same strategy as in the proof of Lemma 7.18. Now we take the coefficient of $a^{j} b^{n-1+i} c^{n-1-j} d^{m-i}$ in (36), obtaining the following equation.

$$
\begin{aligned}
& \left(q^{-n+1}-q^{m}\right) x(m, n-1, i, j)+\left(q^{m-2}-q^{m-n+1}\right) x(m, n-3, i+1, j-1) \\
& +\left(1-q^{m}\right)\left(1-q^{m-1}\right)\left\{x(m-2, n-1, i-1, j)-\left(q^{-2}+q^{m-2}\right) x(m-2, n-3, i, j-1)\right. \\
& \left.+q^{m-4} x(m-2, n-5, i+1, j-2)\right\}-\left(1-q^{m}\right)\{x(m-1, n-1, i, j) \\
& +x(m-1, n-1, i-1, j)-\frac{1+q^{m}}{q}[x(m-1, n-2, i, j-1)+x(m-1, n-2, i, j)] \\
& \left.+q^{m-3}[x(m-1, n-4, i+1, j-1)+x(m-1, n-4, i+1, j-2)]\right\}=0
\end{aligned}
$$

Again it is straightforward to show that this is an identity.

Remark 7.20. As pointed out by one of the referees, the $F_{m}(y)$ are deformation of Al-Salam Chihara polynomials $Q_{m}(x ; a, b \mid q)$. See for example KLS10b, Section 14.8]. To be precise,

$$
F_{m}(y)=(b d)^{m / 2} Q_{m}\left(\frac{b+d}{2 \sqrt{b d}} ; \frac{y a}{\sqrt{b d}}, \frac{y c}{\sqrt{b d}} \mid q\right) .
$$


Thanks to this observation, we can write

$$
F_{m}(y b d)=\frac{\left(y^{2} a b c d ; q\right)_{m}}{(y a)^{m}} \sum_{k=0}^{m} \frac{\left(q^{-m} ; q\right)_{k}(y a d ; q)_{k}(y a b ; q)_{k}}{\left(y^{2} a b c d ; q\right)_{k}(q ; q)_{k}} q^{k}
$$

with $(a ; q)_{k}=\prod_{i=0}^{k-1}\left(1-a q^{i}\right)$. Using this formula, we could give an alternative proof of Proposition 7.16 .

\section{KoORnWinder MOMEnts AT $\xi=q=1$.}

Our goal in this section is to show that when one evaluates Koornwinder moments at $\xi=q=1$, one obtains a beautiful multiplicative formula in terms of hook lengths. This formula is given in Theorem 8.4 below.

To make sense of this specialization, however, we need to be careful. It is clear from Section 3.4 that the Uchiyama-Sasamoto-Wadati solution to the Matrix Ansatz has poles when $q=1$, which is related to the fact that some of our formulas for Koornwinder moments - in particular Theorem 1.1 - are only valid for $q \neq 1$. However, there are other solutions to the Matrix Ansatz which are well-defined at $q=1$. We will review one such solution here, use it to define the partition function and Koornwinder moments (which are the same as our previous definitions, up to a global scalar factor), and then state and prove Theorem 8.4 .

In this section we will abbreviate $K_{\lambda}(1 ; \alpha, \beta, \gamma, \delta ; 1)$ by writing $K_{\lambda}$.

8.1. The Matrix Ansatz at $q=1$. We give now a solution to the Matrix Ansatz at $q=1$, which comes from [USW04, Section 5]. We define tridiagonal matrices $\mathcal{D}$ and $\mathcal{E}$, and vectors $\langle W|$ and $|V\rangle$ by

$$
\begin{gathered}
\mathcal{D}=\left[\begin{array}{cccc}
D_{0}^{\natural} & D_{0}^{\sharp} & 0 & \cdots \\
D_{0}^{b} & D_{1}^{\natural} & D_{1}^{\sharp} & \\
0 & D_{1}^{b} & D_{2}^{\natural} & \ddots \\
\vdots & & \ddots & \ddots
\end{array}\right], \quad \mathcal{E}=\left[\begin{array}{cccc}
E_{0}^{\natural} & E_{0}^{\sharp} & 0 & \cdots \\
E_{0}^{b} & E_{1}^{\natural} & E_{1}^{\sharp} & \\
0 & E_{1}^{b} & E_{2}^{\natural} & \ddots \\
\vdots & & \ddots & \ddots
\end{array}\right], \\
\langle W|=(1,0,0, \cdots), \quad| V\rangle=(1,0,0, \cdots)^{T},
\end{gathered}
$$

where

$$
\begin{array}{ll}
D_{n}^{\natural}=\frac{\alpha+\delta+n(\alpha \beta+2 \alpha \delta+\gamma \delta)}{(\alpha+\gamma)(\beta+\delta)}, & E_{n}^{\natural}=\frac{\beta+\gamma+n(\alpha \beta+2 \beta \gamma+\gamma \delta)}{(\alpha+\gamma)(\beta+\delta)}, \\
D_{n}^{\sharp}=\frac{\alpha}{\alpha+\gamma}[(n+1)(x+n)]^{1 / 2}, & E_{n}^{\sharp}=\frac{\gamma}{\alpha+\gamma}[(n+1)(x+n)]^{1 / 2}, \\
D_{n}^{b}=\frac{\delta}{\beta+\delta}[(n+1)(x+n)]^{1 / 2}, & E_{n}^{b}=\frac{\beta}{\beta+\delta}[(n+1)(x+n)]^{1 / 2},
\end{array}
$$

with

$$
x=\frac{\alpha+\beta+\gamma+\delta}{(\alpha+\gamma)(\beta+\delta)}
$$


We now use the above solution to the Matrix Ansatz to define the partition function and Koornwinder moments at $q=\xi=1$.

We define the following notation:

$$
S=\frac{(\alpha+\gamma)(\beta+\delta)}{\alpha \beta-\gamma \delta}
$$

Definition 8.1. When $q=1$, we define the partition function of the ASEP by $Z_{N}=S^{N}\left\langle W\left|(\mathcal{D}+\mathcal{E})^{N}\right| V\right\rangle 3^{3}$ And we define the Koornwinder moment $K_{\lambda}$ by (11), after setting $\xi=1$ and using the above definition of $Z_{N}$.

Let $\mathcal{C}=\left(c_{i j}\right)$ be the tridiagonal matrix defined by

$$
\begin{aligned}
c_{i, i+1} & =1 \\
c_{i, i} & =S\left(D_{i}^{\natural}+E_{i}^{\natural}\right)=S(x+2 i) \\
c_{i, i-1} & =S^{2}\left(D_{i-1}^{b}+E_{i-1}^{b}\right)\left(D_{i-1}^{\sharp}+E_{i-1}^{\sharp}\right)=S^{2} i(x-1+i) .
\end{aligned}
$$

Lemma 8.2. We have that $Z_{N}=\left\langle W\left|\mathcal{C}^{N}\right| V\right\rangle$.

Proof. Note that for every step from height $i$ to height $i+1$ in a Motzkin path, there must be a corresponding step from height $i+1$ to height $i$ in the Motzkin path. It follows that $\left\langle W\left|\mathcal{C}^{N}\right| V\right\rangle=S^{N}\left\langle W\left|(\mathcal{D}+\mathcal{E})^{N}\right| V\right\rangle$.

Proposition 8.3. We have that

$K_{(N-r, 0,0, \ldots, 0)}=S^{N-r}\left(\begin{array}{c}N \\ r\end{array}\right) \prod_{i=r}^{N-1}(x+i)=\frac{1}{(\alpha \beta-\gamma \delta)^{N-r}}\left(\begin{array}{c}N \\ r\end{array}\right) \prod_{i=r}^{N-1}(\alpha+\beta+\gamma+\delta+i(\alpha+\gamma)(\beta+\delta))$,

where there are precisely $r$ 's in $(N-r, 0,0, \ldots, 0)$.

Proof. If we apply Theorem 4.6 and use the fact that each $c_{i, i+1}=1$, we get

$$
\mathcal{C} \operatorname{Motz}(N, r)=\left\langle W\left|\mathcal{C}^{N}\right| V^{r}\right\rangle=K_{(N-r, 0,0, \ldots, 0)}
$$

But now note that there is a simple recurrence for partial Motzkin paths:

$$
\begin{aligned}
\mathcal{C} \operatorname{Motz}(N, r)= & \mathcal{C} \operatorname{Motz}(N-1, r) c_{r, r}+\mathcal{C} \operatorname{Motz}(N-1, r-1) c_{r-1, r}+\mathcal{C} \operatorname{Motz}(N-1, r+1) c_{r+1, r} \\
= & \mathcal{C} \operatorname{Motz}(N-1, r) S(x+2 r)+\mathcal{C} \operatorname{Motz}(N-1, r-1)+ \\
& \mathcal{C} \operatorname{Motz}(N-1, r+1) S^{2}(r+1)(x+r) .
\end{aligned}
$$

This implies the result.

\footnotetext{
${ }^{3}$ We have included the constant $S^{N}$ in the definition of the partition function so that this definition of $Z_{N}$ equals the specialization of the fugacity partition function $Z_{N}(\xi)$ from Definition 3.8 at $\xi=q=1$. This follows from [USW04, Equation 6.16] and [CSSW12, Theorem 4.1].
} 
8.2. Koornwinder moments at $q=\xi=1$. We identify each partition $\lambda=$ $\left(\lambda_{1}, \ldots, \lambda_{n}\right)$ with its Young diagram, namely a left-justified array of cells with $n$ rows and $\lambda_{i}$ cells in the $i$ th row for $1 \leq i \leq n$. For each cell $z$ of the Young diagram in position $(i, j)$, the hook $H_{\lambda}(z)$ is the set of cells $(a, b)$ such that $a=i$ and $b \geq j$, or $a \geq i$ and $b=j$. The hook length $h_{\lambda}(z)$ is the cardinality of $H_{\lambda}(z)$.

Theorem 8.4 is the main result of this section.

Theorem 8.4. For any partition $\lambda=\left(\lambda_{1}, \ldots, \lambda_{n}\right)$, we have

$$
K_{\lambda}=S^{|\lambda|} \prod_{z \in \lambda}(x+h(z)-1) \cdot \prod_{i=1}^{n-1} \prod_{j=i+1}^{n} \frac{\left(x+\lambda_{i}-\lambda_{j}+j-i-1\right)\left(\lambda_{i}-\lambda_{j}+j-i\right)}{(x+j-i-1)(j-i)} .
$$

To prove Theorem 8.4, we need a few lemmas.

Lemma 8.5. Let $\lambda=\left(\lambda_{1}, \ldots, \lambda_{m}\right)$ and $\nu=\left(\lambda_{1}, \ldots, \lambda_{m}, 0\right)$. Then

$$
K_{\nu}=K_{\lambda} \cdot \prod_{i=1}^{m} \frac{\left(x+\lambda_{i}+m-i\right)\left(\lambda_{i}+m-i+1\right)}{(x+i-1)(i)} .
$$

Proof. By Corollary 5.2 and Proposition 8.3 , we have that

$$
K_{\nu}=\operatorname{det}\left(M_{i j}\right)_{i, j=1}^{m+1}
$$

where

$$
M_{i j}=S^{\nu_{i}+j-i}\left(\begin{array}{c}
\nu_{i}+m+1-i \\
m+1-j
\end{array}\right) \prod_{\ell=m+1-j}^{\nu_{i}+m-i}(x+\ell)
$$

Note that

$$
M_{i j}=S^{\lambda_{i}+j-i}\left(\begin{array}{c}
\lambda_{i}+m+1-i \\
m+1-j
\end{array}\right) \prod_{\ell=m+1-j}^{\lambda_{i}+m-i}(x+\ell)
$$

for $i \neq m+1$, and

$$
M_{m+1, j}=S^{j-m+1}\left(\begin{array}{c}
0 \\
m+1-j
\end{array}\right)= \begin{cases}1 & \text { if } j=m+1 \\
0 & \text { otherwise }\end{cases}
$$

Therefore

$$
\operatorname{det}\left(M_{i j}\right)_{i, j=1}^{m+1}=\operatorname{det}\left(M_{i j}\right)_{i, j=1}^{m}
$$

Meanwhile we have that

$$
K_{\lambda}=\operatorname{det}\left(M_{i j}^{\prime}\right)_{i, j=1}^{m}
$$

where

$$
M_{i j}^{\prime}=S^{\lambda_{i}+j-i}\left(\begin{array}{c}
\lambda_{i}+m-i \\
m-j
\end{array}\right) \prod_{\ell=m-j}^{\lambda_{i}+m-i-1}(x+\ell)
$$


Clearly $M_{i j}^{\prime}=\frac{(m+1-j)(x+m-j)}{\left(\lambda_{i}+m+1-i\right)\left(x+\lambda_{i}+m-i\right)} \cdot M_{i j}$. Therefore

$$
\begin{aligned}
K_{\lambda} & =\operatorname{det}\left(\frac{(m+1-j)(x+m-j)}{\left(\lambda_{i}+m+1-i\right)\left(x+\lambda_{i}+m-i\right)} \cdot M_{i j}\right)_{i, j=1}^{m} \\
& =\frac{\prod_{j=1}^{m}(m+1-j)(x+m-j)}{\prod_{i=1}^{m}\left(\lambda_{i}+m+1-i\right)\left(x+\lambda_{i}+m-i\right)} \operatorname{det}\left(M_{i j}\right)_{i, j=1}^{m} \\
& =\frac{\prod_{j=1}^{m}(j)(x+j-1)}{\prod_{i=1}^{m}\left(\lambda_{i}+m+1-i\right)\left(x+\lambda_{i}+m-i\right)} K_{\nu} .
\end{aligned}
$$

This proves the lemma.

Lemma 8.6. Let $\lambda=\left(\lambda_{1}, \ldots, \lambda_{n}\right)$ and $\nu=\left(\lambda_{1}+1, \ldots, \lambda_{n}+1\right)$. Then

$$
K_{\nu}=K_{\lambda} S^{n} \cdot \prod_{i=1}^{n}\left(x+\lambda_{i}+n-i\right) .
$$

Proof. By Corollary 5.2 and Proposition 8.3 , we have that

$$
K_{\nu}=\operatorname{det}\left(M_{i j}\right)_{i, j=1}^{n}
$$

where

$$
M_{i j}=S^{\lambda_{i}+1+j-i}\left(\begin{array}{c}
\lambda_{i}+1+n-i \\
n-j
\end{array}\right) \prod_{\ell=n-j}^{\lambda_{i}+n-i}(x+\ell) .
$$

Meanwhile we have that

where

$$
K_{\lambda}=\operatorname{det}\left(M_{i j}^{\prime}\right)_{i, j=1}^{n}
$$

$$
M_{i j}^{\prime}=S^{\lambda_{i}+j-i}\left(\begin{array}{c}
\lambda_{i}+n-i \\
n-j
\end{array}\right) \prod_{\ell=n-j}^{\lambda_{i}+n-i-1}(x+\ell) .
$$

We can now write

$$
\begin{aligned}
M_{i j} & =S^{\lambda_{i}+1+j-i}\left(\left(\begin{array}{c}
\lambda_{i}+n-i \\
n-j
\end{array}\right)+\left(\begin{array}{c}
\lambda_{i}+n-i \\
n-j-1
\end{array}\right)\right) \prod_{\ell=n-j}^{\lambda_{i}+n-i}(x+\ell) \\
& =S M_{i j}^{\prime}\left(x+\lambda_{i}+n-i\right)+M_{i, j+1}^{\prime} \frac{x+\lambda_{i}+n-i}{x+n-j-1} .
\end{aligned}
$$

But now since the determinant is alternating in the columns of the matrix $\left(M_{i j}\right)$, we have that

$$
\begin{aligned}
K_{\nu} & =\operatorname{det}\left(M_{i j}\right)=\operatorname{det}\left(S M_{i j}^{\prime}\left(x+\lambda_{i}+n-i\right)\right)_{i, j=1}^{n} \\
& =S^{n} \operatorname{det}\left(M_{i j}^{\prime}\right)_{i, j=1}^{n} \prod_{i=1}^{n}\left(x+\lambda_{i}+n-i\right) \\
& =S^{n} K_{\lambda} \prod_{i=1}^{n}\left(x+\lambda_{i}+n-i\right) .
\end{aligned}
$$

This proves the lemma. 
Proof of Theorem 8.4. We start with a partition $\lambda=\left(\lambda_{1}, \ldots, \lambda_{n}\right)$. We will use induction on both $n$ and $\lambda_{n}$. If $n=0$ then $K_{\lambda}=1$ and we are done. Otherwise if $\lambda_{n}=0$, we apply Lemma 8.5 and get

$$
\begin{aligned}
K_{\lambda} & =K_{\left(\lambda_{1}, \ldots, \lambda_{n-1}\right)} \cdot \prod_{i=1}^{n-1} \frac{\left(x+\lambda_{i}+n-1-i\right)\left(\lambda_{i}+n-i\right)}{(x+i-1)(i)} \\
& =K_{\left(\lambda_{1}, \ldots, \lambda_{n-1}\right)} \cdot \prod_{i=1}^{n-1} \frac{\left(x+\lambda_{i}-\lambda_{n}+n-1-i\right)\left(\lambda_{i}-\lambda_{n}+n-i\right)}{(x+n-i-1)(n-i)}
\end{aligned}
$$

By induction, we know that

$$
K_{\left(\lambda_{1}, \ldots, \lambda_{n-1}\right)}=S^{|\lambda|} \prod_{z \in \lambda}(x+h(z)-1) \cdot \prod_{i=1}^{n-2} \prod_{j=i+1}^{n-1} \frac{\left(x+\lambda_{i}-\lambda_{j}+j-i-1\right)\left(\lambda_{i}-\lambda_{j}+j-i\right)}{(x+j-i-1)(j-i)}
$$

and therefore

$$
K_{\lambda}=S^{|\lambda|} \prod_{z \in \lambda}(x+h(z)-1) \cdot \prod_{i=1}^{n-1} \prod_{j=i+1}^{n} \frac{\left(x+\lambda_{i}-\lambda_{j}+j-i-1\right)\left(\lambda_{i}-\lambda_{j}+j-i\right)}{(x+j-i-1)(j-i)} .
$$

Finally if $\lambda_{n}>0$, we apply Lemma 8.6,

$$
K_{\lambda}=K_{\left(\lambda_{1}-1, \ldots, \lambda_{n}-1\right)} \cdot S^{n} \cdot \prod_{i=1}^{n}\left(x+\lambda_{i}+n-i\right) .
$$

It is easy to see that

$$
\prod_{z \in \lambda}(x+h(z)-1)=\prod_{i=1}^{n}\left(x+\lambda_{i}+n-i\right) \cdot \prod_{z \in\left(\lambda_{1}-1, \ldots, \lambda_{n}-1\right)}(x+h(z)-1) .
$$

By induction, we know that the theorem holds for $K_{\left(\lambda_{1}-1, \ldots, \lambda_{n}-1\right)}$, and therefore we get the desired result for $K_{\lambda}$.

We now explain why Conjecture 4.4 is true when $\xi=q=1$. First note that by iterating Lemmas 8.5 and 8.6 , we can prove the following result.

Proposition 8.7. Let $\nu=\left(\lambda_{1}+1, \ldots, \lambda_{m}+1,0, \ldots, 0\right)$ and $\lambda=\left(\lambda_{1}, \ldots, \lambda_{m}, 0, \ldots, 0\right)$, where there are $r$ 's at the end of each partition. Then

$$
K_{\nu}=K_{\lambda} \cdot \prod_{i=1}^{m} \frac{\lambda_{i}+m+r+1-i}{\lambda_{i}+m+1-i}\left(x+\lambda_{i}+m+r-i\right) .
$$

Corollary 8.8. Conjecture 4.4 is true when $\xi=q=1$, namely the Koornwinder moments at this specialization are polynomials in $\alpha, \beta, \gamma, \delta$ with positive coefficients.

Proof. Note that we can repeatedly apply Proposition 8.7 to an arbitrary partition $\left(\lambda_{1}, \ldots, \lambda_{n}\right)$, so as to express $K_{\lambda}$ in terms of $K_{(0,0, \ldots, 0)}$. It follows from the definition that $K_{(0,0, \ldots, 0)}$ is equal to 1 , and applying the proposition preserves the property of being a polynomial with positive coefficients. 


\section{REFERENCES}

[And83] M. Andréief. Note sur une relation entre les intégrales définies des produits des fonctions. Mém. de la Soc. Sci. de Bordeaux, 2:1-14, 1883.

[AW85] Richard Askey and James Wilson. Some basic hypergeometric orthogonal polynomials that generalize Jacobi polynomials. Mem. Amer. Math. Soc., 54(319):iv+55, 1985.

[BC14] Alexei Borodin and Ivan Corwin. Macdonald processes. Probab. Theory Related Fields, 158(1-2):225-400, 2014.

[Can17] Luigi Cantini. Asymmetric simple exclusion process with open boundaries and Koornwinder polynomials. Ann. Henri Poincaré, 18(4):1121-1151, 2017.

[CMW17] Sylvie Corteel, Olya Mandelshtam, and Lauren Williams. Combinatorics of the twospecies ASEP and Koornwinder moments, 2017.

[CSSW12] S. Corteel, R. Stanley, D. Stanton, and L. Williams. Formulae for Askey-Wilson moments and enumeration of staircase tableaux. Trans. Amer. Math. Soc., 364(11):6009-6037, 2012.

[CW11] Sylvie Corteel and Lauren K. Williams. Tableaux combinatorics for the asymmetric exclusion process and Askey-Wilson polynomials. Duke Math. J., 159(3):385-415, 2011.

[CW13] Sylvie Corteel and Lauren K. Williams. Erratum to "Tableaux combinatorics for the asymmetric exclusion process and Askey-Wilson polynomials" [mr2831874]. Duke Math. J., 162(15):2987-2996, 2013.

[dB55] N. G. de Bruijn. On some multiple integrals involving determinants. J. Indian Math. Soc. (N.S.), 19:133-151 (1956), 1955.

[DEHP93] B. Derrida, M. R. Evans, V. Hakim, and V. Pasquier. Exact solution of a 1D asymmetric exclusion model using a matrix formulation. J. Phys. A, 26(7):1493-1517, 1993.

[GR04] George Gasper and Mizan Rahman. Basic hypergeometric series, volume 96 of Encyclopedia of Mathematics and its Applications. Cambridge University Press, Cambridge, second edition, 2004. With a foreword by Richard Askey.

[GV85] Ira Gessel and Gérard Viennot. Binomial determinants, paths, and hook length formulae. Adv. in Math., 58(3):300-321, 1985.

[Hai] Mark Haiman. Personal communication, January 2007.

[KLS10a] Roelof Koekoek, Peter A. Lesky, and René F. Swarttouw. Hypergeometric orthogonal polynomials and their q-analogues. Springer Monographs in Mathematics. SpringerVerlag, Berlin, 2010. With a foreword by Tom H. Koornwinder.

[KLS10b] Roelof Koekoek, Peter A. Lesky, and René F. Swarttouw. Hypergeometric orthogonal polynomials and their q-analogues. Springer Monographs in Mathematics. SpringerVerlag, Berlin, 2010. With a foreword by Tom H. Koornwinder.

[KM59a] Samuel Karlin and James McGregor. Coincidence probabilities. Pacific J. Math., 9:11411164, 1959.

[KM59b] Samuel Karlin and James McGregor. Coincidence properties of birth and death processes. Pacific J. Math., 9:1109-1140, 1959.

[Koo92] Tom H. Koornwinder. Askey-Wilson polynomials for root systems of type BC. In Hypergeometric functions on domains of positivity, Jack polynomials, and applications (Tampa, FL, 1991), volume 138 of Contemp. Math., pages 189-204. Amer. Math. Soc., Providence, RI, 1992.

[Kra99] C. Krattenthaler. Advanced determinant calculus. Sém. Lothar. Combin., 42:Art. B42q, 67 pp. (electronic), 1999. The Andrews Festschrift (Maratea, 1998).

[Lin73] Bernt Lindström. On the vector representations of induced matroids. Bull. London Math. Soc., 5:85-90, 1973. 
[Mac95] I. G. Macdonald. Symmetric functions and Hall polynomials. Oxford Mathematical Monographs. The Clarendon Press, Oxford University Press, New York, second edition, 1995. With contributions by A. Zelevinsky, Oxford Science Publications.

[MGP68] J Macdonald, J Gibbs, and A Pipkin. Kinetics of biopolymerization on nucleic acid templates. Biopolymers, 6, 1968.

[NNSY01] Jun Nakagawa, Masatoshi Noumi, Miki Shirakawa, and Yasuhiko Yamada. Tableau representation for Macdonald's ninth variation of Schur functions. In Physics and combinatorics, 2000 (Nagoya), pages 180-195. World Sci. Publ., River Edge, NJ, 2001.

[Rai] Eric Rains. Personal communication, June 2013.

[Spi70] Frank Spitzer. Interaction of Markov processes. Advances in Math., 5:246-290 (1970), 1970.

[Sta] Dennis Stanton. Personal communication, January 2016.

[Uch08] Masaru Uchiyama. Two-species asymmetric simple exclusion process with open boundaries. Chaos Solitons Fractals, 35(2):398-407, 2008.

[USW04] Masaru Uchiyama, Tomohiro Sasamoto, and Miki Wadati. Asymmetric simple exclusion process with open boundaries and Askey-Wilson polynomials. J. Phys. A, 37(18):4985$5002,2004$.

[vD95] J. F. van Diejen. Commuting difference operators with polynomial eigenfunctions. Compositio Math., 95(2):183-233, 1995.

[Vie] Gérard Viennot. Une théorie combinatoire des polynomes orthogonaux. Lecture Notes LACIM, UQAM, Montréal, 219 pages, 1984.

[Vie85] Gérard Viennot. A combinatorial theory for general orthogonal polynomials with extensions and applications. In Orthogonal polynomials and applications (Bar-le-Duc, 1984), volume 1171 of Lecture Notes in Math., pages 139-157. Springer, Berlin, 1985.

Laboratoire d'Informatique Algorithmique: Fondements et Applications, Centre National de la Recherche Scientifique et Université Paris Diderot, Paris 7, Case 7014, 75205 Paris Cedex 13 France

E-mail address: corteel@liafa.univ-paris-diderot.fr

Department of Mathematics, University of California, Berkeley, Evans Hall RoOM 913, Berkeley, CA 94720

E-mail address: williams@math.berkeley.edu 